\title{
Functional Anatomy of Macaque Striate Cortex. V. Spatial Frequency
}

\author{
Roger B. H. Tootell, ${ }^{1}$ Martin S. Silverman, ${ }^{2, a}$ Susan L. Hamilton, ${ }^{1}$ Eugene Switkes, ${ }^{3}$ and Russell L. De Valois ${ }^{1}$ \\ 'Department of Psychology, University of California, Berkeley, California 94720, 2Department of Physiology, University of \\ California, San Francisco, California 94143, and ${ }^{3}$ Committee on Psychobiology, Division of Natural Sciences, University of \\ California, Santa Cruz, California 95064
}

\begin{abstract}
When macaque monkeys view achromatic, sinusoidal gratings of a singe spatial frequency, the pattern of ${ }^{14} \mathrm{C}-2-d e o x y-$ $d$-glucose (DG) uptake produced by the gratings is shown to depend on the spatial frequency chosen. When a relatively high (5-7 cycles/deg) spatial frequency is shown binocularly at systematically varied orientations, uptake in parafoveal striate cortex is highest between the cytochrome oxidase blobs (that is, in the interblobs) in layers 1, 2, and 3 . In layers $4 B, 5$, and 6 , where the cytochrome oxidase blobs are faint or absent, DG uptake is highest in a periodic pattern that lies in register with the interblobs of layers $2+3$. When the grating is, instead, of relatively low (1-1.5 cycles/deg) spatial frequency, DG uptake is highest in the blobs, in the blobaligned portions of layers 1-4B, and in the lower-layer blobs as well. These variations in DG topography are confirmed in stimulus comparisons within a single hemisphere. Presumably, this shift in functional topography within the extragranular layer is the primate homolog of "spatial frequency columns" shown earlier in the cat (Tootell et al., 1981; Silverman, 1984).
\end{abstract}

In the well-differentiated architecture of primate striate cortex, laminar differences produced by high- versus lowspatial-frequency gratings are visible as well. Gratings of very high spatial frequency produce much higher uptake in $4 \mathrm{Cb}$ (which receives input from the parvocellular LGN layers) than in $4 \mathrm{Ca}$ (which gets its input from the magnocellular LGN layers). Gratings of low spatial frequency produce the converse result. Presumably, cells in the magnocellular LGN layers and/or in the magnocellular-dominated layer 4Ca have lower average spatial frequency tuning (larger receptive fields) than their counterparts in the parvocellular LGN and/ or in striate layer $\mathbf{4 C b}$.

The DG patterns produced by various spatial frequencies also vary with eccentricity, in a manner consistent with known, eccentricity-dependent variations of receptive-field size and spatial frequency tuning. Thus, gratings of a "middle"-spatial-frequency range (4-5 cycles/deg) produce high uptake in the blobs near the foveal representation and high uptake in the interblobs at more peripheral eccentricities, including

\footnotetext{
Received June 22, 1987; revised Nov. 9, 1987; accepted Nov. 11, 1987.

This work was supported by United States Public Health Service Grants EY00014 and EY-02050 and National Science Foundation Grant BNS 82-02275.

Correspondence should be addressed to Roger B. H. Tootell at his present address: Department of Neurobiology, Harvard Medical School, 25 Shattuck St., Boston, MA 02115.

${ }^{a}$ Present address: Central Institute of the Deaf, $818 \mathrm{~S}$. Euclid Ave., and the Department of Anatomy and Neurobiology, Washington University, St. Louis, MO 63110.

Copyright (C) 1988 Society for Neuroscience $0270-6474 / 88 / 051610-15 \$ 02.00 / 0$
}

$5^{\circ}$. This shift in DG topography also includes the transition zone near $3^{\circ}$, where the level of stimulus-driven uptake is as high in the blob regions as it is in interblob regions. Variations in uptake between layers $4 \mathrm{Ca}$ and $4 \mathrm{Cb}$, as a function of eccentricity, shift in parallel with the changes in the upperlayer topography.

Since the late $1960 \mathrm{~s}$, there has been evidence that the human visual system responds selectively to the spatial frequency of grating patterns (Campbell and Robson, 1968; Blakemore and Campbell, 1969), just as it does to the orientation of grating patterns (Campbell and Kulikowski, 1966; Gilinski, 1968). This psychophysically measured spatial frequency selectivity is presumed to reflect underlying cortical mechanisms, since it shows binocular transfer and is orientation-specific (Blakemorc and Campbell, 1969).

Some early models suggested that the visual system's spatial frequency selectivity would provide a basis for coding images by (global) Fourier analysis (e.g., Kabrinsky, 1966). However, subsequent physiological and psychophysical evidence has not supported this idea. A number of more recent models of early visual processing, such as those described by Marcelja (1980), Daugman (1980), Watson (1983), Wilson and Gelb (1984), and Klein and Levi (1985), use local spatial frequency filters that are more similar to the observed receptive-field properties of cells in the primate visual system. These more recent models have also been successful in describing results from a variety of detection (Watson, 1983; Watson and Ahumada, 1983), discrimination (Wilson, 1985), and hyperacuity (Klein and Levi, 1985; Wilson, 1988) experiments.

In an effort to follow up these findings physiologically, there have been a number of single-unit analyses of the response to sine wave gratings in the striate cortex of monkeys and cats (Maffei and Fiorentini, 1973; De Valois et al., 1975; Schiller et al., 1976; Albrecht, 1978; Movshon et al., 1978c; De Valois et al., 1982). These studies show that most striate cells are reasonably selective for spatial frequency. At least in simple cells, the spatial frequency selectivity is determined in a straightforward way by the receptive-field structure of those cells (Albrecht, 1978; Movshon et al., 1978a; Kulikowski and Bishop, 1981; Mulliken et al., 1984; but see also Schiller et al., 1976). Those simple cells with the narrowest ( $\sim 0.5$ octave) spatial frequency tuning have been found to contain up to 10 alternating excitatory and inhibitory subregions (Mulliken et al. 1984; De Valois et al., 1985). The more numerous simple cells with broader spatial frequency tuning (average, $\sim 1.4$ octaves) have correspondingly fewer subregions. In one report, spatial frequency tuning has also been related to the receptive-field substructure of individual 
complex cells as well (Movshon et al., 1978b). The peak and bandwidth of spatial frequency tuning in complex cells are similar to those found in simple cells (e.g., De Valois et al., 1982).

Cells are more selective for variations in spatial frequency than for variations in the width of bars, edges, or gratings of other waveforms (Schiller et al., 1976; Albrecht et al., 1980; Stork et al., 1982). This follows naturally from a consideration of the receptive-field structure, at least in the case of simple cells. This is one reason for using sine wave gratings in studies of striate cortical function.

It has also been shown that a broad range of "preferred" spatial frequencies is found in any one given region of striate cortex, just as it is found for "preferred" orientations in a given cortical region (Schiller et al., 1976; Movshon et al., 1978a; De Valois et al., 1982). Furthermore, there have been DG and electrophysiological reports that, in the cat, striate cells with similar spatial frequency peaks are grouped together in columns (Tootell et al., 1981; Silverman, 1984), as is the case for orientation and ocular dominance. The fact that both spatial frequency and orientation tuning first appear (or are greatly sharpened) in striate cortex, together with the evidence for a columnar organization of both variables, supports the idea that striate cortex is performing a well-organized orientation-by-spatial frequency analysis of stimuli in each retinotopically restricted portion of the visual field.

If "spatial frequency" columns are a critical component of the visual machinery in striate cortex, and not just some peculiarity (or misperception) of the cat visual system, then presumably they would exist in the well-organized visual system of the monkey, as well as in the cat. In order to investigate this possibility, we have used ${ }^{14} \mathrm{C}$-2-deoxy- $d$-glucose (DG) to label neural activity in response to sinusoidal gratings of various spatial frequencies. Here we report striking evidence for a columnar organization of spatial frequency peak tuning in primate striate cortex.

Other evidence suggests that one might expect laminar variations in striate cortical spatial frequency sensitivity in addition to the columnar variations described above. As early as in the retina, there is evidence that dendritic field size (Perry et al., 1984) and receptive-field size (de Monasterio and Gouras, 1975) are larger for ganglion cells projecting to the magnocellular LGN cells than for ganglion cells projecting to parvocellular LGN cells. In the LGN, parvocellular cells have smaller receptivefield center sizes (Derrington and Lennie, 1984) and somewhat higher spatial resolution (Kaplan and Shapley, 1982; Hicks et al., 1983; Sherman et al., 1984) than magnocellular cells. Since the magnocellular and parvocellular layers project to different sublayers of striate cortex, the receptive-field size in different striate cortical layers might be expected to differ correspondingly. In fact, electrophysiological studies have reported such differences in geniculostriate input layers and beyond (Blasdel and Fitzpatrick, 1984; Livingstonc and Hubcl, 1984). In the present study, we describe differences in the DG sensitivity of various layers (including $4 \mathrm{Ca}$ versus $4 \mathrm{Cb}$ ) to sine wave gratings, which we attribute (at least in part) to differences in the receptive-field size of cells.

Of course, the spatial frequency tuning of a given cell reflects other aspects of its receptive-field organization in addition to simply size. For instance, receptive-field center size determines the upper, but not lower, spatial frequency limit. The lower limit is determined in large part by the shape and strength of the inhibitory surround. Because the receptive-field surround of retinal and LGN is relatively weak, these cells have fairly broad, low-pass spatial frequency tuning; both cells with large and small receptive fields will respond all the way down to the lowest spatial frequencies. In striate cortex, the inhibitory surrounds of most cells are stronger and shaped differently; thus most striate cortical cells respond to a narrower range of spatial frequencies. Because the increased weighting and the reshaping of striate cortical surrounds do not appear to occur within a single synapse, it seems likely that the bandwidth of spatial frequency tuning might vary in different striate cortical layers, or in different proportions of the same layer. In this study we present DG evidence for such a possibility.

There is one additional dimension of striate architecture along which one might expect a variation in spatial frequency peak tuning. Mean receptive-field size is smallest at the foveal representation, becoming progressively larger at increasing eccentricities (Hubel and Wiesel, 1974; Dow et al., 1981; Van Essen et al., 1984). Because of the close relationship between receptivefield width and preferred spatial frequency, it would not be surprising to find electrophysiological evidence for a parallel shift in preferred spatial frequency with changes in eccentricity (Schiller et al., 1976; Movshon et al., 1978b). Again, by coupling DG with sinusoidal gratings of known spatial frequency, we were able to demonstrate a shift in spatial frequency tuning with eccentricity, using DG techniques. Some of these data have been presented in preliminary form (Tootell et al., 1982).

\section{Materials and Methods}

Details of the surgery and histological processing used are similar to those presented earlier (see first paper in this series: Tootell et al., 1988a). In sterile conditions and under full surgical anesthesia, macaque monkeys ( $M$. assamensis, $M$. arctoides, and $M$. cynomolgus) were implanted with plastic headsets about a week prior to the main DG experiment. This eliminated the need for ear bars during the main experiment. Later, for the main experiment, monkeys were anesthesized, paralyzed, and prepared as for electrophysiological recording. An intravenous line was available for the injection of various drugs, and the monkeys were artificially respirated through endotracheal tubes. Either one or both eyes were refracted and optically arranged so that the foveal projection(s) was focused on the center of the stimulus screen. Three millimeter artificial pupils were carefully centered on the optic axis. In the present study, great care was taken to optimize optical factors because some of the stimuli used in such studies (e.g., a 7 cycle/deg grating) are rendered essentially invisible by refractive errors as small as 1 diopter (Westheimer, 1964), or by minor corneal clouding. Stimuli were presented on a Tektronix color monitor driven by a NOVA $4 \mathrm{X}$ computer and a modified Lexidata image processor. Achromatic sinusoidal gratings were produced by modulating the voltage to the 3 color guns in parallel, along axes that were linearly related to luminance rather than to voltage. In more than half of the cases, multiunit activity that was recorded from foveal striate cortex was used both to locate the foveal projection in the visual field and as a measure of binocular correspondence. In some cases, the initial optical refraction was fine-tuned by measuring the response of foveal single units to variations in supplementary trial lenses. DG $(12.5-50 \mu \mathrm{Ci} / \mathrm{kg})$ was injected while the monkeys viewed each stimulus. Protracted injections (up to $10 \mathrm{~min}$ ) were used in order to prevent inadvertent stimulus biases in the pattern of DG uptake. The specific stimuli used in this study are described individually in Results and in Table 1 of Tootell et al., 1988a. In describing results from the present study, we refer to case numbers from that table. After $30-45 \mathrm{~min}$, monkeys were euthanized and transcardially perfused with a $10 \%$ sucrose phosphate-buffered solution, given a light formalin fixation, and then rinsed again with the phosphate-buffered solution. The brain was removed from the skull, and the opercular portion of striate cortex (representing eccentricities from $0^{\circ}$ to about $8^{\circ}$ ) was dissected free from the rest of the brain. The tissue was laid surface-down on a glass slide, and frozen while flat. Cryostat sections from this block were cut exactly parallcl with the opcrcular cortical surfacc. Subsequent autoradiographs were exposed at $-70^{\circ} \mathrm{C}$ in order to preserve the cytochrome oxidase 


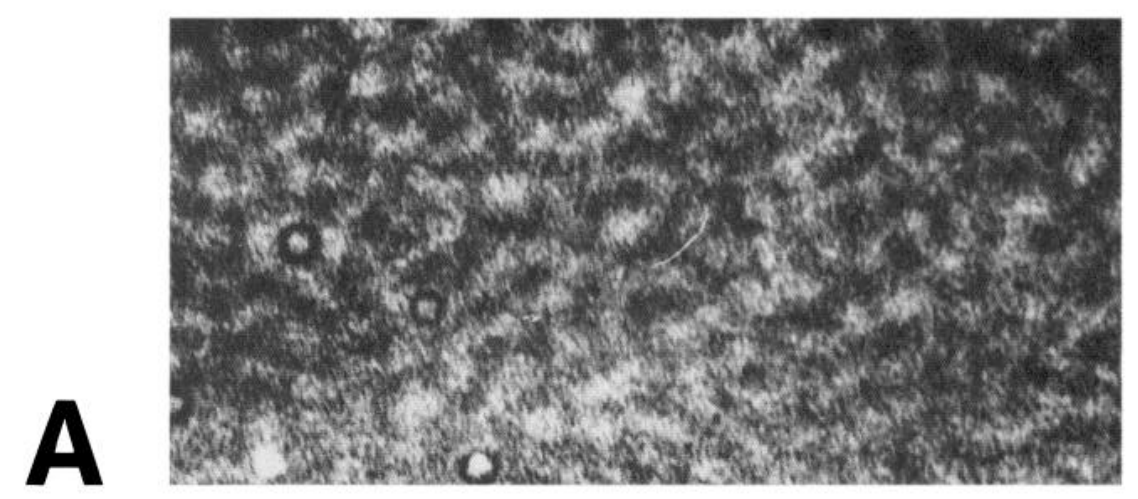

Figure 1. A, Deoxyglucose autoradiograph from a monkey presented with a high- ( 6.5 cycles/deg) spatial-frequency grating at varied orientations, viewed binocularly. The stimulus was moved at a range of speeds, in both directions. The autoradiograph is produced by a section through layer 3 of striate cortex, cut tangential to the flattened cortical surface, spanning the representation of eccentricities from about $4^{\circ}$ to $5^{\circ}, B$, The same section after staining for cytochrome oxidase. The regions of high DG uptake (dark regions of $A$ ) lie in the regions of low cytochrome oxidase activity (light regions of $B$ ). The geometrical relationship of the 2 patterns (from a slightly different portion of the same section) is shown more clearly in Figure 2, $A, B$. Calibration bar, $2.5 \mathrm{~mm}$.

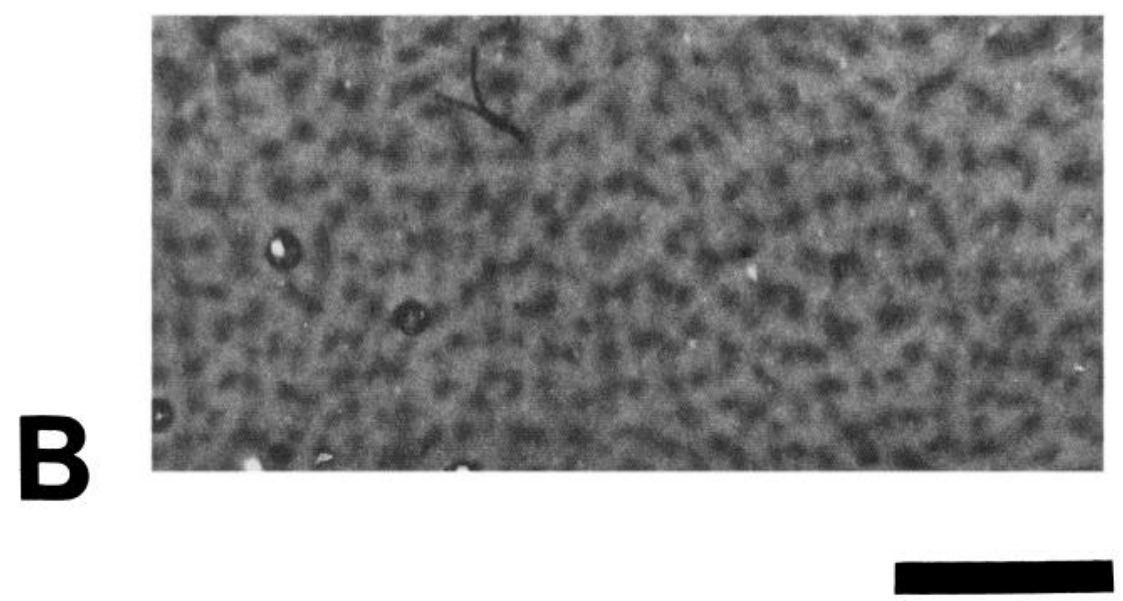

histochemistry of the tissue (after Humphrey and Hendrickson, 1983). Most of the tissue was exposed along with ${ }^{14} \mathrm{C}$ radiographic standards so that relative uptake levels could be quantified. After autoradiography, sections were stained for cytochrome oxidase (cytox) or (less commonly) Nissl substance.

The main probe stimulus used in these experiments was a sinusoidal grating of a single spatial frequency (e.g., cases 19 and 20). Another 9 macaques were shown sinusoidal grating stimuli in which the spatial frequency of the grating was different in different halves or quarters of the stimulus (split-field stimuli) (cases 24, 27, 29, 30, 36, 39, 40, 44, 49). In some of these 9 cases, one hemisphere was devoted to other stimulus comparisons, described in related studies. Another 3 macaques were shown stimuli in which the spatial frequency of a square wave or sine wave grating was systematically varied during the DG infusion (cases $37,46,50$ ). In all the above cases, orientation was systematically varied, and the stimulus was presented binocularly. In order to eliminate the possibility of artifacts due to stimulation at a single nonzero disparity, we sometimes varied the binocular disparity over a small range during periods of DG uptake. All stimuli described in this section were achromatic, and grating contrast was set between 70 and $100 \%$.

In a number of tissue sections, image-processing techniques were used to quantify the topographical relationship between regions of high DG uptake and cytox activity. In those cases, both the autoradiography and the cytox-stained sections were digitized and then digitally filtered to emphasize local contrast and remove both noise and gradual variations in optical density. A binary threshold applied to the filtered images allowed their dichotomization into DG-active or inactive and cytoxactive or inactive regions. Since autoradiography and cytox staining were carried out sequentially on the same tissue sections, these patterns could be precisely superimposed. The extent of overlap of the DG and cytox patterns could thus be quantitatively assessed and readily visualized when superimposed in different colors on a color monitor (Switkes et al., 1986).

Choice of spatial frequency. The distribution of peak spatial frequen- cies in single units measured at any one eccentricity is unimodal, with the number of cells preferring higher and lower frequencies falling off smoothly on either side of the mode. The distribution of peak spatial frequencies is thus unlike the distribution of peak orientations: the latter is quite flat.

In the cat, it has been hard to demonstrate DG spatial frequency columns by stimulating with middle spatial frequencies (e.g., 0.75 cycle/ $\mathrm{deg}$ ) because of this nonuniform distribution of peak spatial frequencies, and because cells tuned to spatial frequencies near the mean of the distribution actually respond over a significant portion of the total range. The successful approach has been instead to stimulate with spatial frequencies that are either very low (i.e., $0.25 \mathrm{cycles} / \mathrm{deg}$ ) or very high (i.e., 1.8 cycles/deg) relative to the distribution of peak frequencies at foveal and parafoveal eccentricities in the cat (Tootell et al., 1981; Silverman, 1984). Presumably, stimulation with spatial frequencies at the extremes of the distribution is more effective in functionally isolating cells because the spatial frequency bandpass of the underlying low- or high-frequency population is effectively only half as wide as when stimulating with middle spatial frequencies.

In monkey striate cortex, the distribution of peak spatial frequencies is qualitatively similar, but quantitatively shifted to higher frequencies: at eccentricities from $3^{\circ}$ to $5^{\circ}$ (where we have concentrated our DG analysis), the mean is about 3 cycles/deg, and the range extends from about 0.5 to 10 cycles/deg (De Valois et al., 1982). In order to functionally isolate monkey striate cells that are tuned to low or high spatial frequencies, we usually stimulated with spatial frequencies chosen from the extremes of the underlying distribution of neural sensitivities, as was done earlier in the cat. To isolate cells tuned to low spatial frequencies, we used sinusoidal gratings of $1-1.5$ cycles/deg, and, for high spatial frequencies, gratings of 6.0-7.5 cycles/deg. In order to examine the variation in spatial frequency sensitivity with eccentricity, we stimulated with middle spatial frequencies (3-4.5 cycles/deg) in a few instances. Because spatial frequency sensitivity in striate cortex varies with eccentricity, we use the terms "low," "middle," and "high" spatial 
frequencies not as constants, but in relationship to the frequency range of cells at a given eccentricity.

\section{Results}

\section{Columnar variations}

In the first paper in the present series (Tootell et al., 1988a), we showed that binocular stimulation with a grating of high contrast and systematically varied orientation and spatial frequency produces a relatively uniform pattern of uptake within all layers of parafoveal striate cortex. Thus, if stimulation with a grating of a single spatial frequency (but otherwise identical characteristics) produces a systematically different pattern of uptake, then that would constitute preliminary evidence for the existence of a spatial frequency-specific cortical architecture.

We first describe results from animals stimulated with highspatial-frequency gratings. In one representative case (case 19), a monkey was binocularly stimulated with a sinusoidal grating of $6.5 \mathrm{cycles} / \mathrm{deg}$ at systematically varied $\left(45^{\circ} \mathrm{steps}\right)$ orientations moved at a range of velocities and in a direction that was reversed every $5 \mathrm{sec}$. The binocular convergence was varied by $0.5^{\circ}$ on either side of apparent zero disparity during the period of DG uptake.

The result of such stimulation is shown in Figure $1 A$. This autoradiograph is taken from layer 3, at a representation of about $3^{\circ}-5^{\circ}$ eccentricity. The autoradiographic topography is strikingly annular, or doughnut-shaped, in design. When the autoradiograph and the corresponding cytox-stained section were carefully aligned on a light box, the sums of the 2 patterns appeared to cancel each other, as if they were topographically inverse.

In order to quantify this rather surprising result, digital imageprocessing techniques were used to compare the topographies of the cytox-active regions and the DG uptake (Switkes et al., 1986). A color-coded representation of this comparison is found in Figure $2 A$. Regions of high DG uptake are color-coded red, and regions of high cytox activity are color-coded green. Regions corresponding to both high DG uptake and high cytox activity were rendered yellow. In Figure $2 A$, the DG and cytox images are aligned correctly, and in Figure $2 B$ the 2 patterns have been shifted slightly (and randomly) relative to each other. There is obviously much less overlap (yellow) in Figure $2 A$ than in $2 B$ : the DG patterns are concentrated in the interblob regions much more than would be expected by chance. The interblob bias in DG uptake can also be confirmed by simple inspection of Figure $2 A$. The annulus-shaped topography can also be seen in deoxyglucose autoradiographs in layers $2,3,4 \mathrm{~B}, 5,6$, and perhaps 1 and $4 \mathrm{Ca}$. Details of these DG patterns in different laminae and at different eccentricities are described more fully below.

The characteristic pattern of high interblob uptake in response to a high-spatial-frequency grating is significant for 2 reasons. First, a pattern of DG uptake that is systematically highest in the interblob regions in response to any visual stimulus has never been described before; all other stimuli produce a pattern of uptake in which DG is either highest in the blobs or relatively equal in blob and interblob regions (Horton and Hubel, 1981; Tootell et al., 1982; Humphrey and Hendrickson, 1983; Horton, 1984). The pattern of high interblob uptake is thus a striking illustration of the extent to which DG and cytochrome oxidase measures can be dissociated. Second, the annulus-shaped DG topography can be seen in all extragranular layers, and the topographic arrays in different laminae are in vertical registration. We are thus looking at a generally columnar pattern of DG uptake; control experiments (described below) lead us to believe that, in Figure 1, we have labeled columns of cells tuned to high spatial frequencies (relative to a given eccentricity) in monkey visual cortex.

If cells that are tuned to high spatial frequencies are in fact organized in columns running vertically through the interblob regions, then it is logical to suppose that cells at the other end of the functional continuum (that is, tuned to low spatial frequencies) would be located at the other end of the physical continuum (in the blobs).

This was tested by showing a different monkey (case 20) a low-spatial-frequency grating ( $1.0 \mathrm{cycle} / \mathrm{deg})$, at all orientations (in $45^{\circ}$ steps), which was moved at a range of velocities in a direction that was reversed every $5 \mathrm{sec}$. The stimulation was binocular and, again, binocular convergence was varied over a small range during the period of DG uptake.

In layer 3, the DG topography produced by this stimulus was strikingly bloblike, rather than "annulus-like" as in the highfrequency cases. A closeup of the DG topography, and of the corresponding cytox-stained section, is shown in Figure 3. The contrast of the DG pattern in Figure 3 is somewhat lower than in other cases stimulated identically. As in the previous case, the autoradiograph and section are taken from about $3^{\circ}-4^{\circ}$ retinal eccentricity.

From inspection of Figure 3, it appears that the DG is highest in the blobs, rather than between the blobs. This was confirmed by quantitative comparison, using digital image processing (see Fig. $2 C$ ). The bloblike DG topography produced by low-spatialfrequency stimulation occurs in layers $2,3,4 \mathrm{~A}, 4 \mathrm{~B}, 5,6$, and perhaps layer 1 . In all layers, the blob-shaped regions of high uptake lie in vertical registration. Thus the pattern of high uptake produced by a low-spatial-frequency grating is basically columnar, as is the pattern produced by a high-spatial-frequency grating. However, as is obvious from the relationship of each pattern to the cytochrome oxidase blobs, the low- and highspatial-frequency DG columns are topographically complementary to one another.

Given different receptive fields that contain the same number of subregions or show the same degree of bandpass spatial frequency selectivity, cells with lower-spatial-frequency tuning will correspond to larger receptive fields, while those with higherfrequency tuning will have smaller receptive fields. Thus, the present results strongly suggest that the receptive fields of cclls in the blobs are larger than those in the interblobs. This is entirely consistent with results from electrophysiological mapping studies (Hubel and Livingstone, 1987).

In our experiments, a given visual stimulus produces a similar pattern of DG uptake in different animals. Interanimal variability is certainly not large enough to produce an artifactual shift of maximum DG uptake all the way from the blobs to the interblobs. Nevertheless, to be absolutely certain that these DG effects were really the result of variations in spatial frequency, rather than in some other, uncontrolled variable, we simultaneously presented both high and low spatial frequencies, in different portions of the visual field, to the same animal.

In one very informative case (39), we divided the stimulus screen along the horizontal meridian. In the upper half, we presented a sinusoidal grating of low $(0.9 \mathrm{cycle} / \mathrm{deg})$ spatial frequency. In the lower half, a grating of medium-high ( 4.4 cycle/ deg) spatial frequency was presented. Both gratings were drifted at a range of temporal frequencies $(1-5 \mathrm{~Hz}$ ), and thus a range of velocities, across the full range of orientations in $45^{\circ}$ steps. 
The images in the 2 eyes were converged by adjusting Risley prisms, and the binocular disparity was left constant throughout the period of DG uptake.

The results of such a case are shown in Figure 4. This figure includes tissue from the entire lateral surface of the operculum. Figure $4 A$ shows the pattern of DG uptake in layer 3 , and Figure $4 B$ shows the same section after staining for cytochrome oxidase. The representation of the fovea is to the right of Figure $4 \mathrm{~A}$ (starred), and of the parafovea to the far left. The representation of the horizontal meridian (the stimulus border between the gratings of different spatial frequency) can be clearly seen in Figure $4 A$, running close to horizontal, dividing the section into an upper and lower region (between dashed lines, Fig. 4). Between the 2 regions is a narrow strip that was stimulated by the $4.4 \mathrm{cycle} / \mathrm{deg}$ grating in one eye and the $0.9 \mathrm{cycle} / \mathrm{deg}$ grating in the other eye. This strip is thus an artifact produced by slight misalignment of the eyes. It has been ignored in the subsequent discussion.

We first discuss DG differences in the parafoveal representation, since this is the eccentricity from which data have been described thus far. The parafoveal pattern produced by the 4.4 cycle/deg grating (surrounding the "I" in Fig. 4A) looks strikingly annular (that is, highest in the interblobs), and that produced by the 0.9 cycle/deg grating (surrounding the " $\mathrm{B}$," bottom left, Fig. 4A) looks as if it is highest in the blobs. In order to confirm this, the parafoveal DG and cytox patterns from the same section were processed to obtain a quantitative comparison (see Fig. 2D). In Figure 2 it is strikingly clear that the relatively high spatial frequency grating produces high uptake between the blobs, and that the low-spatial-frequency grating produces highest uptake in the blobs themselves.

It is worth pointing out that the representation of the inferior half of the visual field (above the dashed lines in Fig. 4) is significantly greater than that of the superior visual field (below the dashed lines, Fig. 4). This supports other evidence for a superior-inferior anisotropy in the visual-field representation of striate cortex (Van Essen et al., 1984; Tootell et al., 1988b).

\section{Variations with laminae}

In addition to being inversely related in the topographic plane, the patterns of uptake produced by low- versus high-spatial- frequency gratings are also complementary in the laminar domain within layer $4 \mathrm{C}$. Gratings of a high spatial frequency produce DG uptake that is much higher in layer $4 \mathrm{Cb}$ than in $4 \mathrm{Ca}$. Conversely, gratings of a low spatial frequency produce patterns of uptake that are much higher in $4 \mathrm{Ca}$ than in $4 \mathrm{Cb}$.

These 2 DG results can be seen in Figures 5 and 6, respectively. Figure 5 was taken from a case in which a monkey was shown a grating of high $(7 \mathrm{cycle} / \mathrm{deg})$ spatial frequency at all orientations, binocularly. In this animal, uptake is clearly highest in layer $4 \mathrm{Cb}$. It is also high in layer $4 \mathrm{~A}$, which is consistent with other evidence (Hubel and Wiesel, 1972) suggesting that this layer receives LGN input predominantly from the parvocellular, rather than magnocellular, layers. By comparing Figure $5, A$ and $B$, one can again see that DG uptake in layer 3 of this case is highest between the blobs rather than on the blobs.

The case presented in Figure 6 was shown a low- $(1$ cycle/ deg) spatial-frequency grating, again at all orientations and binocularly. Here the uptake is clearly higher in layer 4Ca than in $4 \mathrm{Cb}$.

There is an electrophysiological corrclatc for thesc laminar differences in DG uptake. Blasdel and Fitzpatrick (1984) have reported that receptive fields in layer $4 \mathrm{Ca}$ are significantly larger than those in underlying $4 \mathrm{Cb}$. There are also reports that cells in the magnocellular LGN layers have receptive fields that are larger than those in the parvocellular layers (e.g., Derrington and Lennie, 1984). So it is not surprising that their respective striate targets (4Ca and $4 \mathrm{Cb}$ ) show corresponding differences in receptive-field size and spatial frequency tuning. Inasmuch as spatial frequency tuning and receptive-field size are correlated, the present laminar DG differences in spatial frequency sensitivity are entirely consistent with the receptive-field size differences reported in previous electrophysiological studies. However, it is possible that other functional differences between magno- and parvocellular cells exaggerate this apparent difference between spatial frequency selectivity in DG results from layers $4 \mathrm{Ca}$ and $4 \mathrm{Cb}$.

In addition to this major laminar subdivision in $4 \mathrm{C}$ between the effects of low- and high-spatial-frequency gratings, there are also a number of more subtle laminar differences in layers beyond $4 \mathrm{C}$. These additional laminar variations (as well as other frequency-specific DG variations described below) are most

Figure 2. $A-G$, Patterns in each panel are taken from a section of layer 3, cut parallel with the cortical surface. Regions of high DG uptake are color-coded red, regions of high cytochrome oxidase staining are coded green, and regions of intersecting high DG and high cytox activity are coded yellow. Regions of low DG uptake and low cytox activity appear black. The color coding is done without recourse to hand tracing; there is very little potential for experimenter bias in this form of analysis. $A$ and $B$, Topographical relationship of the cytochrome oxidase blobs to the pattern of DG uptake produced by a high spatial frequency grating at all orientations, viewed binocularly. $A-D$, Representation of parafoveal ( $4^{\circ}-5^{\circ}$ ) eccentricities. In $A$ the cytochrome oxidase and DG patterns are precisely aligned; it can be seen that the regions of high DG lie preferentially off the blobs in the interblob regions. This is made more obvious by shifting the cytox and DG patterns slightly relative to each so that they are now out of alignment. This more random relationship between the DG and cytox is shown in $B$ : there is much more yellow, indicating a much greater intersection of the 2 patterns. $C$, Same as $A$ except that in this case the animal has been stimulated with a sinusoidal grating of a low-spatial frequency. Again, the grating was presented binocularly and at all orientations. The images are taken from a single layer 3 section from parafoveal striate cortex (color coding as described above). In this case it is evident that regions of high DG uptake are generally coextensive with the cytox blobs, although some minor differences appear in these filtered, binarized images. $D$, Split-field comparison of the DG and cytox topographies produced by locally high-versus-low spatial frequencies. In this case the animal was shown a sinusoidal grating of medium-high (4.4 cycle/deg) spatial frequency in one half of the visual field, and a grating of low $(0.9 \mathrm{cycle} / \mathrm{deg})$ spatial frequency in the other half. Both gratings were shown concurrently, binocularly, and at systematically varied orientations and drift speeds. The medium-high spatial frequency grating produced the pattern of DG uptake in the upper half of $D$ (above the double arrows) and the low-spatial frequency grating produced the pattern below the double arrows. The region of $D$ along a horizontal strip between the double arrows received input from both high- and low-spatial frequencies, one from each eye, due to minor misalignment of the eyes. The color coding in this panel is as described above. In the top half of the figure, the regions of high DG uptake (red) systematically avoid the cytochrome oxidase blobs, so that the blobs show up generally as green. Below the double arrows, the regions of high DG uptake fall systematically on the cytochrome oxidase blobs, so that the blobs are generally yellow. $E$, Same as $D$, but taken from the foveal representation of the same tissue section. Abnve the double arrows, the DG pattern was produced by a $4.4 \mathrm{cycle} / \mathrm{deg}$ grating, and below the arrows it was produced by a 0.9 cycle/deg grating. Color coding is as described above. At this eccentricity, 4.4 cycles/deg is a "low" enough spatial frequency to produce high uptake on the blobs, rather than between them. Calibration bar, $2.5 \mathrm{~mm}$. 

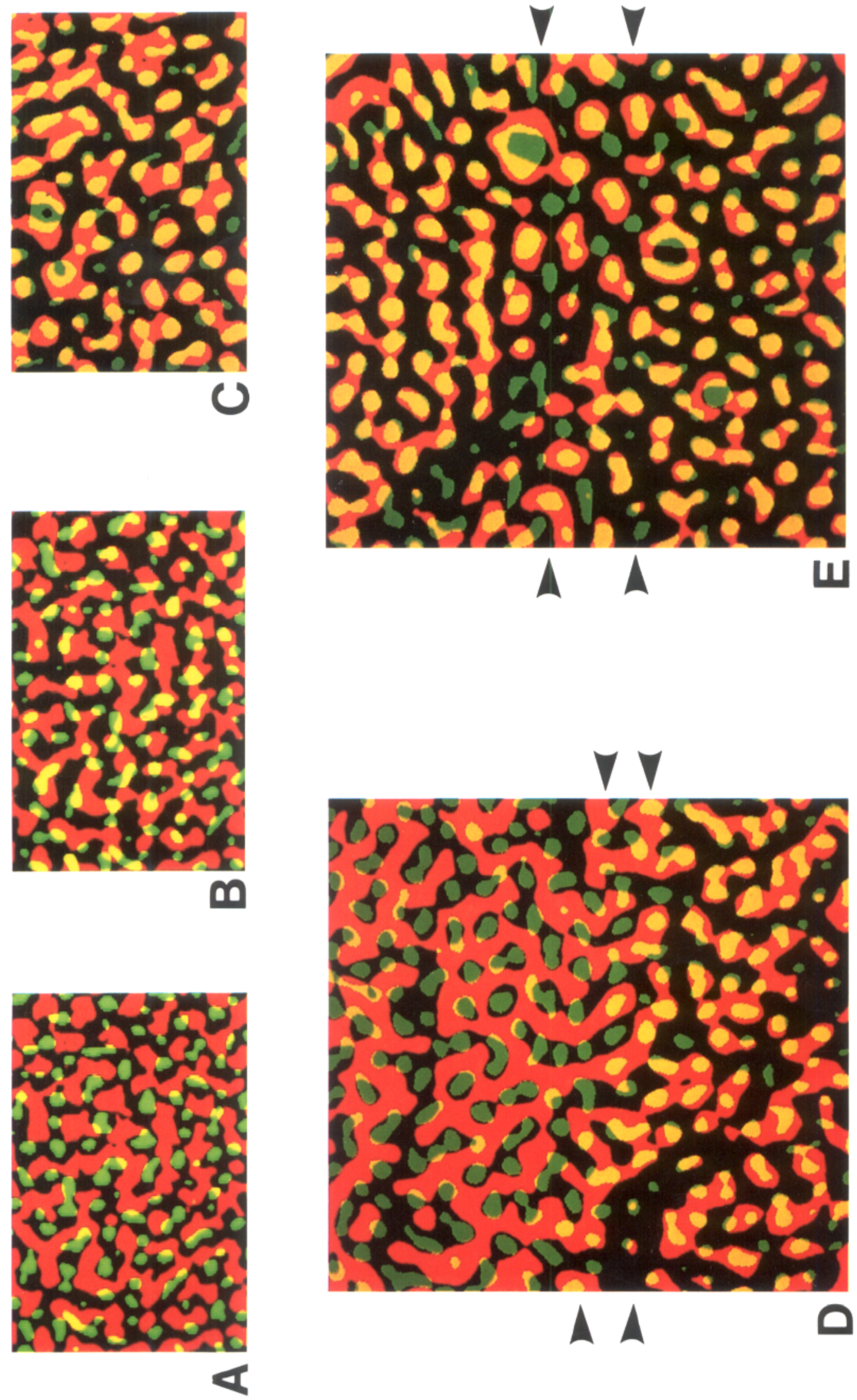

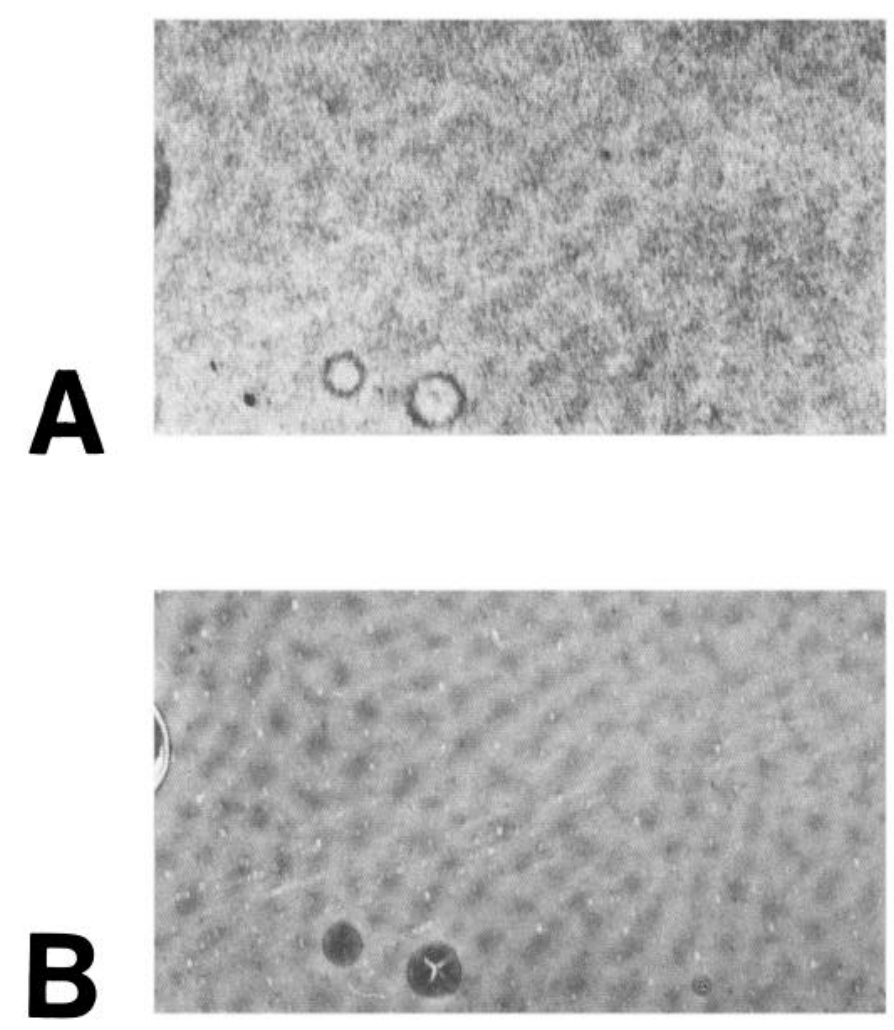

Figure 3. A, Autoradiograph from a monkey presented with a low( 1.5 cycle/deg) spatial-frequency grating at varied orientations, viewed binocularly. Except for the spatial frequency, the stimulus was identical to that used to produce Figure $1 A$. As in Figure 1, the section used to produce Figure $3 A$ was taken from striate layer 3 , from the representation of eccentricities near $4^{\circ}-5^{\circ}$. The pattern of cytochrome oxidase staining in the section producing $2 \mathrm{~A}$ is shown in $2 \mathrm{~B}$. Although the contrast of the DG patterns in this case is fairly light, the areas of highest uptake are in general coextensive with regions of high cytochrome oxidase activity. This is more obvious when both images are color-coded and superimposed (see Fig. 2C). Calibration bar, $2.5 \mathrm{~mm}$.

convincingly demonstrated by data from the animal described above (case 39), which was shown a low- vs a medium-highspatial-frequency grating at the same time in different portions of the visual field (see Fig. 4).

We first confine our observations to laminar differences at parafoveal eccentricities. These are shown in Figure 7. Except for a slightly darker layer $5 \mathrm{~A}$ in this animal, the laminar patterns seen in this case are typical of those produced by low and high spatial frequencies in all other animals, in both split-field and full-field presentations. In Figure 7, $A-I$, uptake produced by the "high"-spatial-frequency grating appears in the top half, and uptake produced by the "low" appears in the bottom half.

In this and other favorable high-frequency cases, the columnar DG periodicites extend into layer 1. DG uptake and the contrast of the annular DG pattern are moderate throughout layers 2 and 5 (see Fig. 7, $B$ and $H$ ). DG contrast becomes abruptly greater in layers 3 and 6 , and the annular topography is more obvious in these layers (Fig. 7, $C-I$ ). Levels of uptake are high in layer $4 \mathrm{~A}$ and moderate in layer $4 \mathrm{~B}$. The annulae are obvious in layer 4B (Fig. 7E), but relatively absent from layer 4A (see Fig. $7 D$ ).

The laminar distribution of uptake in the region stimulated by low spatial frequencies is quite different. The contrast of the low-spatial-frequency bloblike pattern increases gradually from layer 1 through layer $4 \mathrm{~A}$, and uptake becomes slightly darker in layer $4 \mathrm{~B}$ (see Fig. $7 E$ ). The complete lack of laminar transition between layers 3 and $4 \mathrm{~A}$ is unique among binocular DG patterns. It is especially interesting because in other DG patterns that are confined largely to the blobs, layer $4 \mathrm{~A}$ is labeled darkly, without obvious blobs, as it is in the cytochrome oxidase pattern itself (see Tootell et al., 1988c). Another point of interest is that the change in contrast between layers $2+3$ that occurs in highspatial-frequency regions has no analog in the regions stimulated by low spatial frequencies: the contrast of the low-frequency DG blobs is about equal in layers 2 and 3.

In comparisons between different animals, we find major differences between the effects of low- and high-spatial-frequency gratings within layer $4 \mathrm{C}$ (Figs. 5 vs 6 ). In the within-animal comparison illustrated in Figures 4 and 7, these laminar differences in spatial frequency sensitivity are also evident (compare Fig. 7, $F$ and $G$ ). For instance, in the portion of cortex responding to a low- $(0.9 \mathrm{cycle} / \mathrm{deg})$ spatial-frequency grating, uptake is clearly much higher in layer $4 \mathrm{Ca}$ than in $4 \mathrm{Cb}$. In the region stimulated by a medium-high- $(4.4 \mathrm{cycles} / \mathrm{deg})$ spatialfrequency grating, uptake is about equally high in layers $4 \mathrm{Ca}$ and $4 \mathrm{Cb}$. From other cases (e.g., Fig. 5), we know that the balance of uptake in layers $4 \mathrm{Ca}$ versus $4 \mathrm{Cb}$ would have shifted to favoring $4 \mathrm{Cb}$ if we had stimulated with an even higher (e.g., 7 cycle/deg) spatial-frequency grating.

\section{Variations with eccentricity}

Thus far we have considered the topography of DG results at an eccentricity of $3^{\circ}-5^{\circ}$. Because the average spatial frequency peak varies inversely with eccentricity (e.g., Schiller et al., 1976; Movshon et al., 1978a; De Valois et al., 1982; Silverman, 1984), we can expect a change in the topographic pattern of DG uptake with eccentricity as well. A spatial frequency that is "low" relative to the mean in foveal striate cortex will be a "high" spatial frequency more peripherally.

This shift with eccentricity is demonstrated quite dramatically in Figure 4. At eccentricities near $5^{\circ}$, the pattern of DG uptake

\footnotetext{
Figure 4. Within-animal comparison of the DG effects of a low- versus a medium-high-spatial-frequency grating. Deoxyglucose autoradiograph $(A)$ and the corresponding section stained for cytochrome oxidase $(B)$ from a monkey shown gratings of 2 different spatial frequencies in different halves of the visual field. The stimulus consisted of a sinusoidal grating of either $0.9 \mathrm{cycles} / \mathrm{deg}$ (above the horizontal meridian) or $4.4 \mathrm{cycles} / \mathrm{deg}$ (below the horizontal meridian). Both gratings were presented at a variety of drift rates in both directions, at systematically varied orientations. The eyes were converged as well as possible and the stimulus was shown binocularly. The section used to produce $A$ was cut tangential to the flattened cortical surface across the whole lateral operculum. The fovea is represented at the right-most tip of the section (star), and more peripheral regions are represented to the left. The representation of the horizontal meridian (corresponding to the border between the 2 stimulus halves) can be seen running from the lower left to the upper right through the middle of the section, between the dashed lines added outside the section. The visual field is represented in an inverted fashion on striate cortex. The pattern of uptake in the upper half of the section was produced by the 4.4
} 

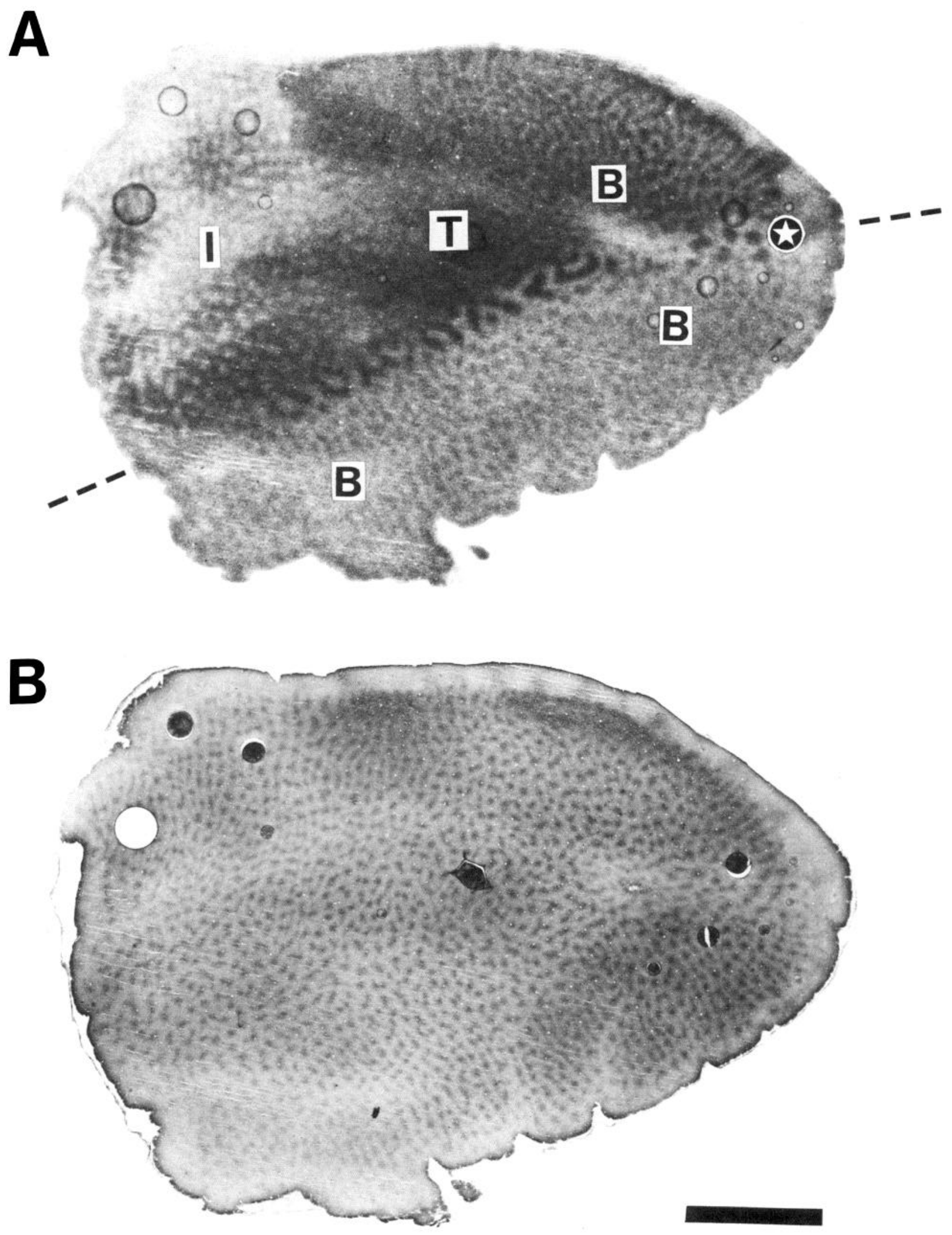

cycles/deg grating, and the lighter, more dotted pattern of uptake in the lower half of the section was produced by the 0.9 cycle/deg grating. The pattern of uptake produced by the $4.4 \mathrm{cycle} / \mathrm{deg}$ grating shifts with eccentricity. At the foveal representation (right-most corner), the regions of high uptake overlay the blobs (see Fig. $2 D$ ), and near $4^{\circ}-6^{\circ}$ eccentricity (top left), the regions of high uptake overlay the interblobs (Fig. $2 E$ ). Between these 2 extremes is a transition region where stimulus-driven DG uptake is high but relatively balanced in blob versus interblob regions (top, center). Where DG periodicities overlay the blobs, this is indicated with a $B$ in $A$. Where the DG overlays the interblobs, this is indicated with $I$. The transition zone is labelled $T$. Calibration bar, $5 \mathrm{~mm}$. 


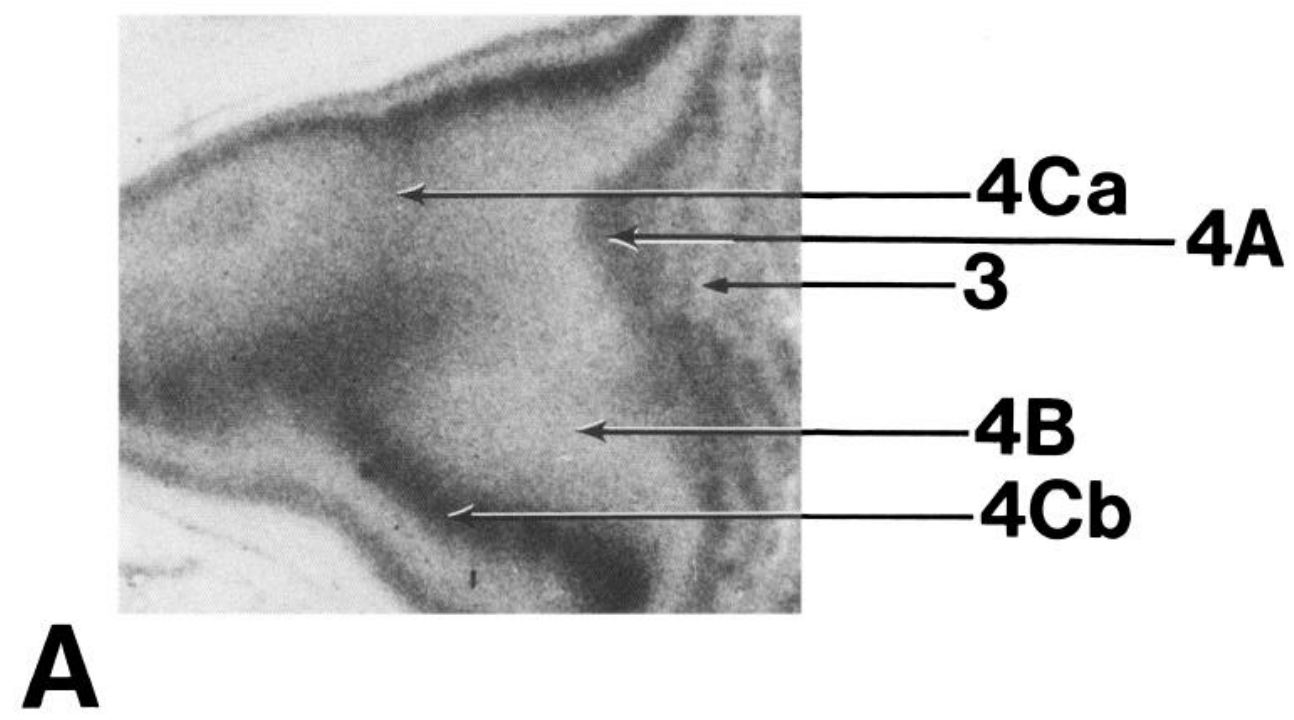

Figure 5. Preferential labeling of layer $4 \mathrm{Cb}$ produced by a high-spatial-frequency grating. Deoxyglucose autoradiograph $(A)$ and corresponding cytoxstained section $(B)$ from an animal shown a high- ( 7 cycle/deg) spatial-frequency grating. The grating was shown binocularly, at varied orientations, and drifted at various drift rates in both directions. The section is cut near-tangential to the underside of the operculum, including layers 3 through $4 \mathrm{Cb}$. Laminar differences in DG uptake are indicated by arrows showing representative portions of each layer; the laminar boundaries can be confirmed by comparison of $B$ to $A$. In response to a high-spatial-frequency grating, uptake in $4 \mathrm{Cb}$ is much higher than that in layer $4 \mathrm{Ca}$. Calibration bar, $5 \mathrm{~mm}$.

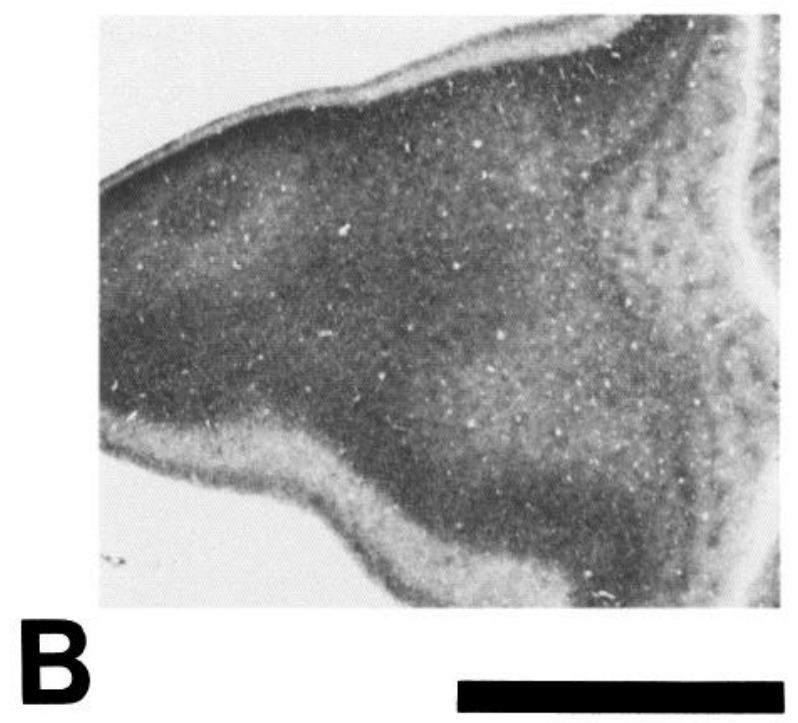

produced by a 4.4 cycle/deg grating (upper half of Fig. $4 A$ ) is highest in the interblobs ("I") (see Fig. $2 D$ ). That is, 4.4 cycles/ deg is clearly a "high" spatial frequency relative to the underlying distribution of single-unit sensitivities at this eccentricity (De Valois et al., 1982). However, near the foveal representation (upper-right-hand “B," Fig. $4 A$ ), the pattern of DG uptake produced by the same spatial frequency is highest in the blobs, as if that spatial frequency were now "low" relative to the mean (see Fig. 2E).

At eccentricities between these 2 extremes, the pattern of DG uptake is approximately equal in blob and interblob areas (in the transition region surrounding "T" in Fig. $4 A$ ). This is to be expected from a "middle" spatial frequency, that is, one that is near the mean of the frequency range at that particular striate eccentricity. A priori, one might naively expect that middle spatial frequencies would instead produce a periodic pattern of DG uptake. However, this has been hard to demonstrate because a large proportion of all striate cells have some sensitivity to middle spatial frequencies, or because there are only 2 classes of spatial frequency sensitivity (magno- and parvo-related), which are both activated by middle frequencies.
In the 4.4 cycle/deg region, the laminar pattern of DG uptake varies in lockstep with the eccentricity-dependent shift in topography from interblobs to blobs. For example, within the striate area stimulated by the $4.4 \mathrm{cycle} / \mathrm{deg}$ grating, uptake in layer $4 \mathrm{Cb}$ (but not $4 \mathrm{Ca}$ ) becomes progressively lighter nearer the foveal representation. By the time the foveal representation is reached, uptake in layer $4 \mathrm{Cb}$ is nearly as light as in the adjacent region stimulated by a much lower $(0.9$ cycle/deg) spatial frequency.

We have seen a similar frequency-eccentricity interaction in the effects of DG uptake (e.g., from interblob to blob with decreasing eccentricity) in all monkeys that were shown spatial frequencies of 6-7 cycles/deg. However, in these cases, the $a b$ solute spatial frequency of the gratings was higher than that shown in Figures 5 and 6 . Thus, in these higher-frequency cases, the overall pattern of DG topography described in Figure 5 is shifted towards the foveal representation. For example, in monkeys stimulated with a 6.5-7 cycle/deg grating, both the regions of uniform uptake and of bloblike uptake occur nearer the foveal representation (and thus are reduced in area) relative to cases stimulated with slightly lower spatial frequencies. 


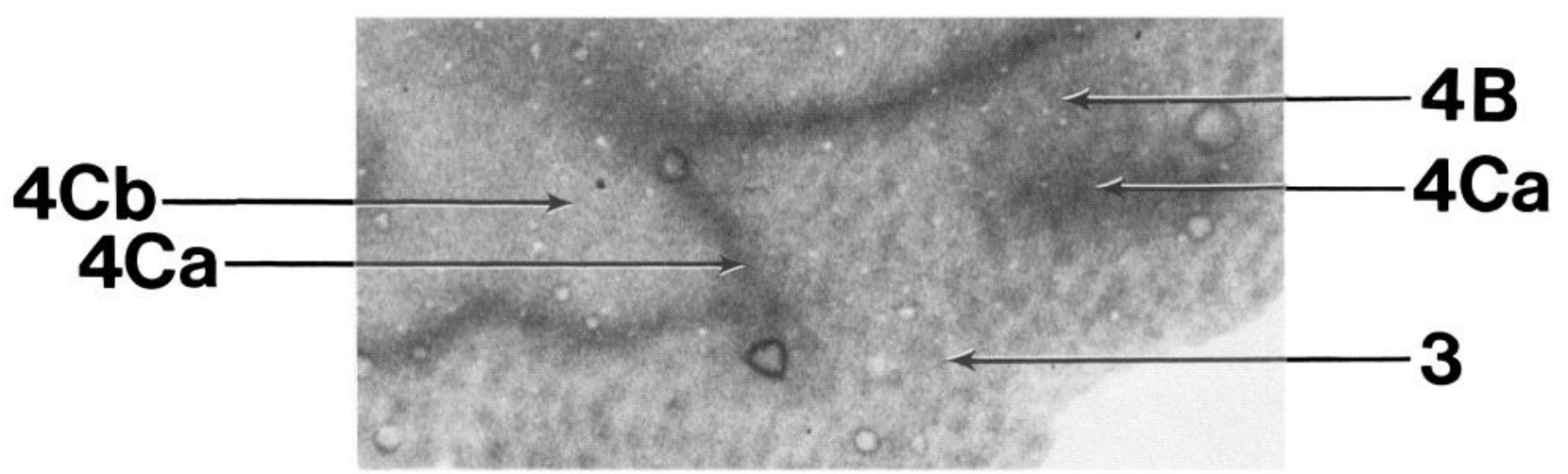

A
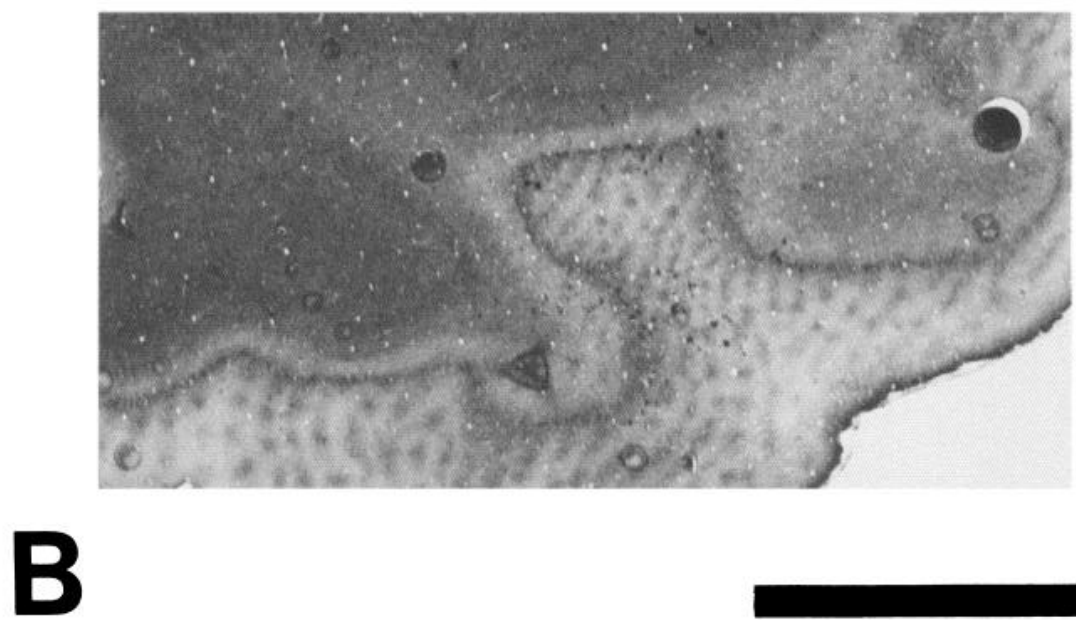

Figure 6. Preferential labeling of layer $4 \mathrm{Ca}$ by a low-spatial-frequency grating. The animal in this case was stimulated binocularly with a low (1 cycle/deg) sinusoidal grating, at various orientations, drifted at a range of drift rates and directions. $A$, The corresponding section after staining for cytochrome oxidase. Representative DG portions from each of the various layers are indicated by arrows in $A$; these can be confirmed by examination of the cytochrome oxidase laminar differences visible in $B$. In response to a low-spatial-frequency grating, uptake in layer $4 \mathrm{Ca}$ is much higher than that in layer $4 \mathrm{Cb}$. Calibration bar, $5 \mathrm{~mm}$.

The shift in average spatial frequency tuning with eccentricity can also be seen in a different aspect of the DG results. Let us assume that we are examining a striate cortical region in which grating frequency " $\mathrm{X}$ " corresponds locally to a "high" spatial frequency, and thus causes maximal DG uptake in the interblobs. If one then moved to regions representing progressively more peripheral eccentricities, one would expect the same grating " $\mathrm{X}$ " to produce progressively less overall uptake, since this spatial frequency would become increasingly too "high" for the underlying cell sensitivities.

Such an eccentricity-dependent shift can be seen in Figure 8, which shows the decrease in uptake, within layer 2 , from a representation of about $3^{\circ}(F)$ to about $8^{\circ}(P)$. This animal (case 19) was shown a drifting, 6.5 cycle/deg grating at varied orientations, binocularly. Uptake is highest in the interblobs throughout, but the level of uptake produced by a single spatial frequency decreases to essentially negligible values with increasing eccentricity.

There also appears to be some inherent variability between animals in the range of underlying sensitivities between eccentricities of $0^{\circ}$ and $6^{\circ}$. For instance, in one exceptional animal (case 40 ), the layer 3 topography produced by the $7 \mathrm{cycle} / \mathrm{deg}$ grating was highest between the blobs at parafoveal eccentricities, and uniform more centrally, without ever quite achieving a clear-cut "blob" topography at the fovea. Such a finding (i.e., 7 cycle/deg is still a relatively high frequency near the fovea) implies a lower sensitivity to high spatial frequencies in the whole animal. This minor variability in the DG results is not particularly surprising, given the variation in spatial frequency sensitivity of primate psychophysical subjects (Ginsburg et al., 1984) and in the effects of minor optical deficits during development (as would occur during undiagnosed myopia or hyperopia during development) (Hendrickson et al., 1987; Kiorpes et al., 1987; Movshon et al., 1987).

\section{Discussion}

These deoxyglucose data indicate quite clearly that low spatial frequencies produce highest uptake on the blobs, and that high spatial frequencies produce highest uptake in the interblob zones. At least 2 models are consistent with this data. One model (the magno-parvo model) assumes that the peak spatial frequency tuning of cells in the blobs is different from that in the interblobs, 


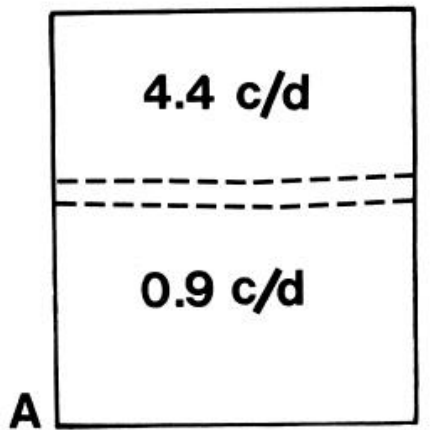

D

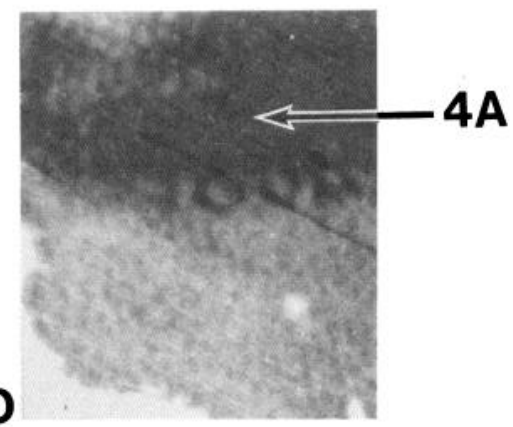

E

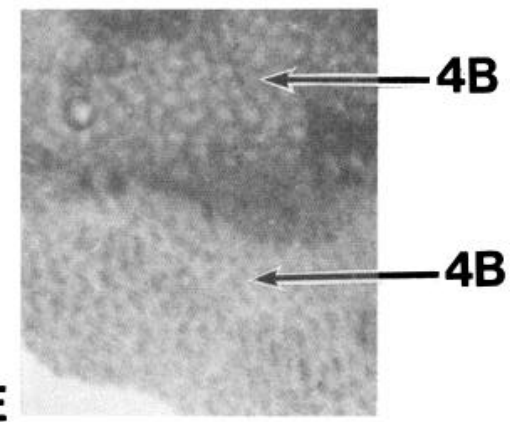

F

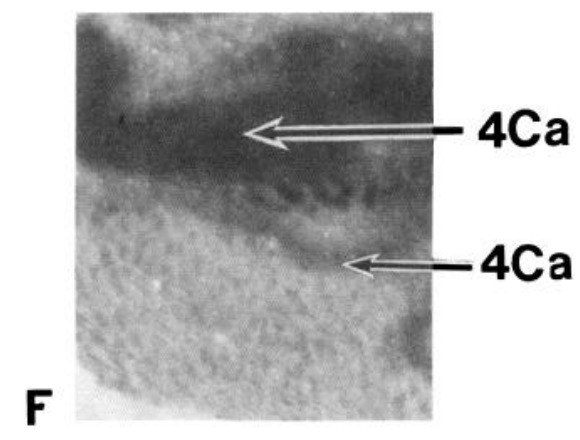

H
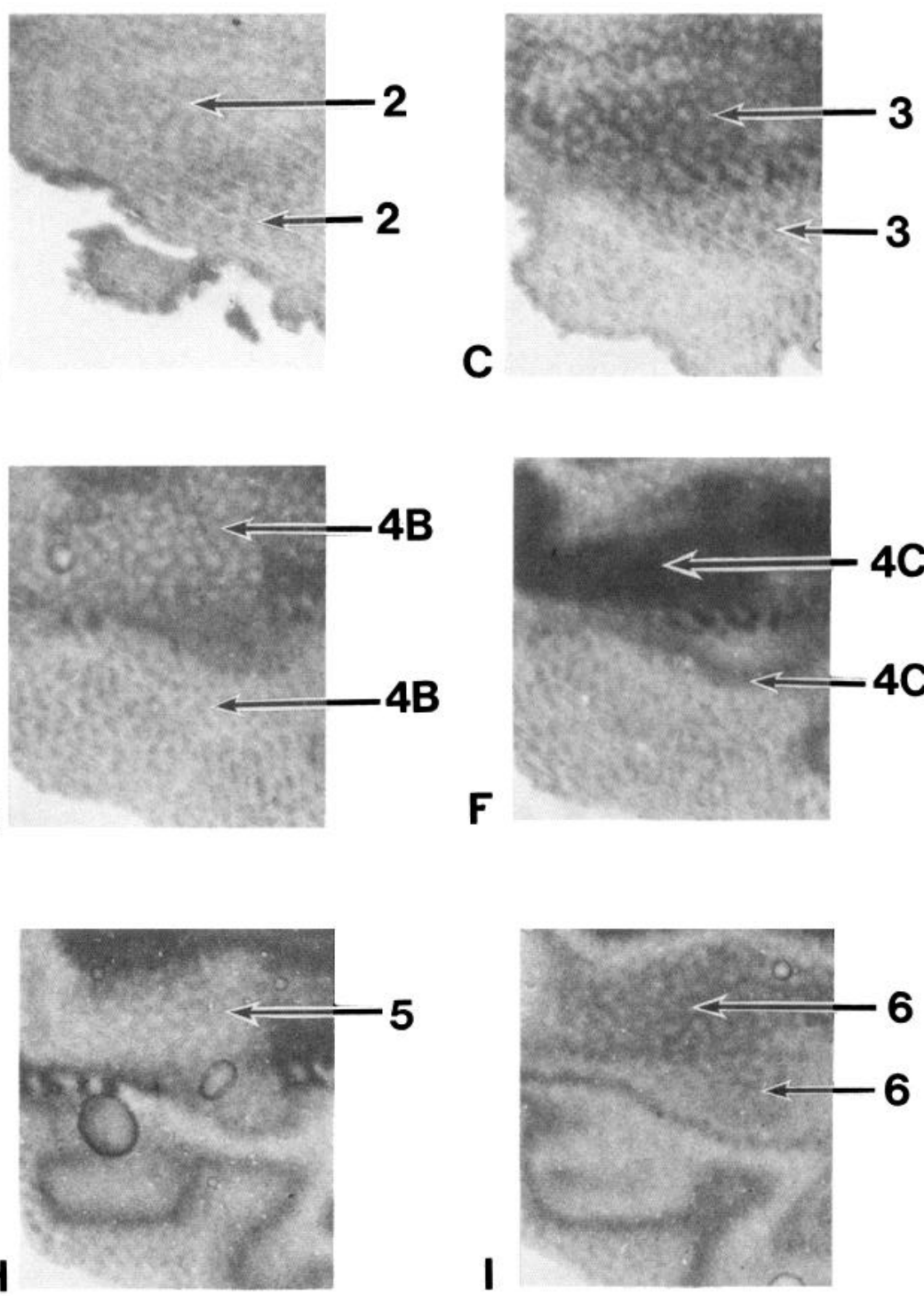

3

B

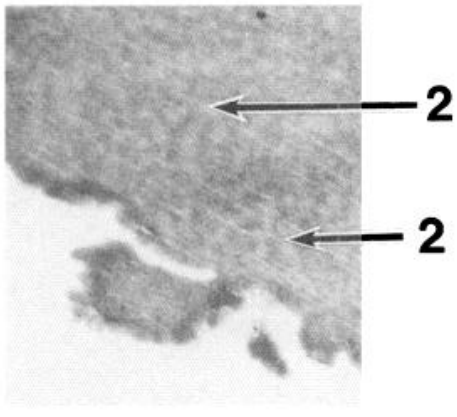

C

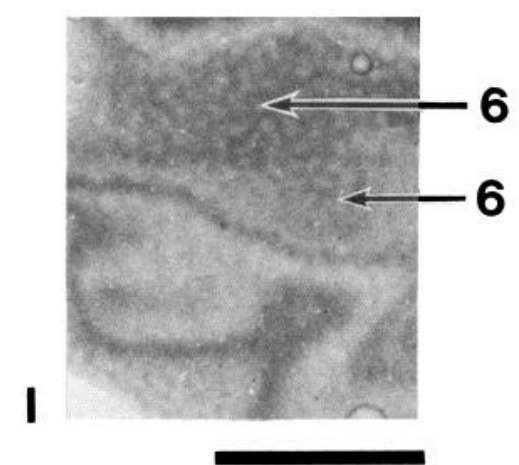

Figure 7. Laminar differences between the DG patterns produced by low- and high-spatial-frequency gratings. The data in $B-I$ are taken from topographically aligned portions of the parafoveal representations of the case illustrated in Figure 4. As diagrammed in $A$, the lower portion of all of the subsequent panels indicates a region stimulated by a low- $(0.9 \mathrm{cycle} / \mathrm{deg})$ spatial-frequency grating, and the upper portion was stimulated by a grating of a spatial frequency $(4.4 \mathrm{cycle} / \mathrm{deg})$ that is relatively high for this eccentricity $\left(4^{\circ}-5^{\circ}\right)$. A thin stripe of mixed activity (due to minor misalignment of the eyes) separates the 2 frequency regions; this is indicated between the 2 dashed lines of $A$. In both frequency regions, patterns of uptake are topographically uniform in layers $4 \mathrm{Ca}$ and $4 \mathrm{Cb}$. In layer $4 \mathrm{Cb}$, uptake is much higher in response to the $4.4 \mathrm{cycle} / \mathrm{deg}$ grating than it is to the $0.9 \mathrm{cycle} / \mathrm{deg}$ grating $(G)$. The spatial frequency of the $4.4 \mathrm{cycle} / \mathrm{deg}$ grating was not high enough to produce light uptake within layer $4 \mathrm{Ca}(F)$. Further laminar distinctions are described in the text. Calibration bar, $5 \mathrm{~mm}$.

but that a more fine-grained organization of spatial frequency tuning does not exist within either compartment. This model further assumes that the differences in spatial frequency tuning of blob and interblob cells are derived from differences in receptive-field center size in magno- and parvocellular LGN cells.

In another model (the continuum model), we assume that striate cortical cells in layers outside of $4 \mathrm{C}$ have a continuous range of peak spatial frequency selectivities and a topographic organization. Those neurons that respond to the locally lowest spatial frequencies would be at the center of the blobs, and those with higher spatial frequency peak progressively further from the blobs.

The present data do not allow us to choose definitively be- tween the 2 models. What we have done instead is to organize the evidence for and against each of the models.

First we will discuss evidence for the magno-parvo model. In the present DG data, when levels of uptake are higher in the blobs than in the interblobs, uptake is invariably higher in $4 \mathrm{Ca}$ than in $4 \mathrm{Cb}$. When the situation is reversed in the blobs and interblobs, it is reversed in layers $4 \mathrm{Ca}$ and $4 \mathrm{Cb}$ as well.

Electrophysiological studies indicate that receptive-field center size is apparently smaller in the parvocellular stream than in the magnocellular stream. This is observed all the way from the retina (Perry et al., 1984) through the LGN (Derrington and Lennie, 1984) and into the geniculorecipient layers of striate cortex (Blasdel and Fitzpatrick, 1984). The electrophysiological 
evidence for smaller receptive fields in $4 \mathrm{Cb}$ than in $4 \mathrm{Ca}$ is strongly supported by DG evidence showing sharper retinotopic borders in $4 \mathrm{Cb}$ than in $4 \mathrm{Ca}$ (Tootell et al., 1988d). It is therefore attractive to suppose that receptive-field size (and spatial frequency tuning) in the blobs would match that of $4 \mathrm{Ca}$, and that the receptive-field size (and spatial frequency tuning) of the interblobs would match that of $4 \mathrm{Cb}$.

Other evidence does not support the magno-parvo model directly, but weighs against the continuum model. For instance, if such a continuum existed, then the interblob regions with the highest peak spatial frequency tuning would presumably be at the furthest point from the blobs, that is, at the intersection of diagonals between any 4 rectangularly packed blobs. Given such an architecture, one should be able to produce a shift in DG topography from an annular, interblob-type pattern to a more spotty subset of that pattern (that is, spots at the intersection of diagonals between the blobs) with increasing eccentricity, in response to a high-spatial-frequency grating. Such a test case is illustrated in Figure 8. Instead of annulae that become increasing spotlike at greater eccentricities, the DG pattern remains annular, but decreases in overall level of uptake at greater eccentricities.

One other piece of evidence is consistent with the magnoparvo model, but counter to the continuum model. In preliminary experiments with voltage-sensitive dyes, it has been straightforward to produce evidence for low-versus high-spatial frequency in blobs versus interblobs, respectively, with optical techniques (Tootell and Blasdel, 1987). However, attempts to produce optical evidence for finer subdivisions of spatial frequency sensitivity within either blob or interblob regions have produced negative results. Since the optical recording data are not subject to the types of resolution limitations of the DG technique, this provides further support for the idea that the spatial frequency "columns" reflect 2 populations of cells (blob and interblob) with different mean spatial frequency tuning, and nothing more.

Other evidence can be offered in support of the continuum model, or that runs counter to the magno-parvo model. We might begin by emphasizing that the observation of only 3 distinct DG patterns (bloblike, uniform, and annular) does not automatically rule out the continuum model. Three such distinct patterns could be a consequence of the rather broad and overlapping tuning functions of striate cortical neurons, and of the fact that more neurons are tuned to the middle-spatial-frequency range. Given the average $\sim 1.4$ octave bandwidths of striate cells (De Valois et al., 1982), a high-spatial-frequency grating would stimulate a limited subset of high-frequency cells and a lowspatial-frequency grating a limited set of low-frequency cells giving annular and blob DG patterns, respectively. However, a middle-spatial-frequency grating would stimulate almost all cells to some extent (particularly at the high contrasts we used) and thus produce the uniform pattern of DG uptake observed in this case.

The impetus for the continuum model arises from the body of psychophysical and physiological literature that has concluded that there are multiple spatial frequency channels for a given region of the visual field (for review, see Braddick et al., 1978, and Shapley and Lennie, 1985). For instance, De Valois et al. (1982) recorded from some 30 striate cortical cells at a single retinotopic locus (lumping together cells from different layers and blob-interblob regions) and found evidence for cells tuned to a variety of different spatial frequencies and orientations.

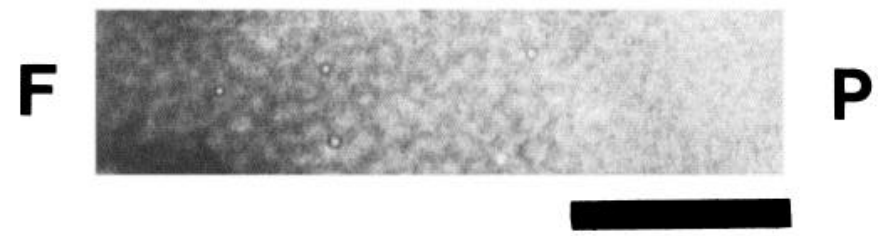

Figure 8. Eccentricity-dependent shift in levels of uptake implies an eccentricity-dependent shift in underlying spatial frequency tuning. The monkey in this case was stimulated (binocularly) with a high- ( 6.5 cycle/ deg) spatial-frequency grating at varied orientations, drift rates, and directions. Shown here is the DG autoradiograph from a section cut parallel with the cortical layers. The section is from the upper layers, and spans the representation of eccentricities from $3^{\circ}$ (far left, $F$ ) to $8^{\circ}$ (far right, $P$ ). Throughout, the periodic patterns of high uptake overlies the interblobs. However, the level of DG uptake decreases markedly at greater eccentricities. This is presumably due to a corresponding decrease in the number of cells responding to spatial frequencies as high as 6.5 cycles/deg. Calibration bar, $5 \mathrm{~mm}$.

Watson and Robson (1981) addressed the same question in a psychophysical experiment using localized Gaussian patches to eliminate the possible confounding factor of eccentricity, and concluded that a minimum of 10 channels at a given locus was required to explain their detection-identification results. It is not yet clear how to reconcile suggestions of 2 spatial frequency channels (from the present DG study) with previous evidence supporting the idea of multiple spatial frequency channels.

Presumably, the spatial frequency tuning of magnocellular and parvocellular cells in the LGN would give some insight into the tuning of their target neurons in striate cortex. Here the evidence stacks up generally against the magno-parvo model: the average peak spatial frequency in magnocellular cells is only about half that of parvocellular cells, and there is a great deal of overlap (Kaplan and Shapley, 1982; Hicks et al., 1983; Derrington and Lennie, 1984). Although magnocellular receptivefield centers are, on average, larger than their parvocellular counterparts, the overall spatial frequency tuning of the 2 types of LGN cells is largely overlapping because (1) the comparatively weak surrounds of both types of LGN cell produce only a very shallow response falloff on the low-spatial-frequency side of the curve, and (2) on the high-spatial-frequency side, the higher contrast sensitivity of the magnocellular cells partially compensates for the smaller receptive-field centers of the parvocellular cells.

It could be that the increased surround strength of striate cortical cells introduces a difference in spatial frequency tuning to magno- and parvo-derived cells based on residual differences in receptive-field center size in the 2 populations. In fact, there is some evidence for this. In the present DG data, and in preliminary electrophysiological data, the bandwidth of spatial frequency tuning appears rather broad in layers $4 \mathrm{Ca} / 4 \mathrm{Cb}$ (but more discrete than in LGN cells) and the tuning appears even more discrete in (at least the interblobs of) layers $2+3$.

One aspect of the present DG results seems to argue against a magno-parvo basis for the observed spatial frequency differences. Striate cortical layer 4B receives little or no input from the parvocellular stream (Lund, 1973; Fitzpatrick et al., 1985; Tootell et al., 1988c, d). However, this layer shows very clear spatial frequency differences in DG uptake that are in register with the blobs and interblobs in other layers. It appears that cells of different peak spatial frequency tuning are being actively segregated in this layer, even though all the cells are receiving 
information primarily or exclusively from magnocellular channels.

An analogous situation may exist to a lesser degree in layers 2 and 3 . The magno-parvo model posits an input to some blob cells from the magnocellular layers, and to all interblob cells from the parvocellular layers, and there is some evidence for this (see above). However, there is also strong circumstantial evidence for a parvocellular-derived input to at least some blob cells (Livingstone and Hubel, 1984; Michaels, 1986, 1987; Tootell et al., 1988c), as well as to those in the interblobs. We cannot yet rule out the possibility that the blob cells that respond well to low-spatial-frequency gratings receive much of their information via the parvocellular LGN layers.

Presumably, unit electrode penetrations near-parallel to the cortical surface through layers $2+3$ would clarify whether there is a more organized continuum of peak tuning. In the monkey, this experiment has not yet been performed, to our knowledge. In the cat, homologous electrode penetrations parallel to the layers have shown periodic oscillations in spatial frequency tuning (Silverman, 1984), and an organized continuum of peak frequencies can be construed from this data. However the oscillations are noisy enough so that it is also possible that the periodic oscillations might reflect 2 groups of cells with different frequency tuning, plus noise.

The variations in DG results with eccentricity may clarify corresponding questions in psychophysics and physiology. It is universally accepted that acuity is highest in the fovea and falls off with eccentricity (e.g., Wertheim, 1894). The maximum spatial frequency that can be resolved falls off with increasing eccentricity as well. Some psychophysical results (e.g., Robson and Graham, 1981) suggest that the maximum sensitivity to low-spatial-frequency gratings also occurs in the fovea, which implies that there is an increase in the range of underlying cell sensitivities near the fovea, as well as an increase in the mean. Other psychophysical results (e.g., Rovamo and Virsu, 1979) suggest that the range does not increase near the fovea; only the mean does. Single-unit data from the cat (Movshon et al., 1978c; Silverman, 1984) and monkey (De Valois et al., 1982) suggest that both the range and the mean increase towards the fovea or area centralis, but the unit data are limited by sample size, variability between animals, and the various vicissitudes of recording. It is thus worth pointing out that the DG data, which are not limited in these ways, also suggest an increase in the range, as well as the mean, of spatial frequency tuning towards the fovea.

These DG data are as follows: First, in all animals stimulated with a low- $(\sim 1$ cycle/deg) spatial-frequency grating, the pattern of uptake is highest in the blobs at all eccentricities between $0^{\circ}$ and $\sim 5^{\circ}$, and the level of uptake does not vary significantly with eccentricity (e.g., Fig. 4). Presumably, this indicates that $\sim 1$ cycle/deg occupies a position that is "equally low" for the distribution of underlying cell sensitivities, all the way from the fovea to $5^{\circ}$ peripheral or so. Second, uptake in response to a higher- ( $\sim-5$ cycles/deg) spatial-frequency grating is highest in the blobs near the fovea, equally high in blobs and interblobs at the representation of about $2^{\circ}-3^{\circ}$, and highest in the interblobs at $5^{\circ}$. Third, uptake levels in response to the 4-5 cycles/deg grating are much higher than those produced by the $1 \mathrm{cycle} / \mathrm{deg}$ grating when the effects of both are compared near the fovea.

The most straightforward interpretation of all these data is that most cells at about $5^{\circ}$ eccentricity lie within the range of 1-5 cycles/deg, whereas those toward the fovea must range from around 1 cycle/deg to much greater than 5 cycles/deg, with a mean near $5 \mathrm{cycles} / \mathrm{deg}$ or slightly higher. According to other DG data, spatial frequencies as high as 10 cycles/deg are necessary to reliably produce high interblob labeling in the foveal representation. Thus the range of spatial frequency sensitivity in the foveal representation appears to be approximately double that found at $5^{\circ}$.

Overall, the spatial frequencies that we assume to be "high" or "low" on the basis of the DG evidence correspond to those that also would be called "high" or "low" on the basis of singleunit reports. At $3^{\circ}-4^{\circ}$ eccentricity, 6.5 cycles/deg is clearly a "high" spatial frequency, relative to single-unit reports from a number of animals (Schiller et al., 1976; De Valois ct al., 1982). Corresponding data from foveal eccentricities are too sparse to judge with confidence, and in these paralyzed animals, the reported peaks can be artifactually depressed because of corneal clouding or other optical limitations. However, we can infer that a spatial frequency of 4-5 cycles/deg would occupy a position in the low-to-medium end of the foveal distribution, on the basis of single-unit responses of awake, behaving animals (in which optical limitations are not a factor) (Poggio et al., 1977; Dow et al., 1981). Judging from the distribution of singleunit peaks, 1 cycle/deg is clearly a "low" spatial frequency at both foveal and parafoveal eccentricities (Schiller et al., 1976; De Valois et al., 1982).

Using spatially diffuse variations in color, or equiluminant color-varying gratings of low to middle spatial frequency, it is also possible to produce high DG uptake on the blobs. Such color-specific uptake correlates well with the electrophysiological data of Livingstone and Hubel (1984), who showed that approximately three-fourths of the cells in the blobs respond well to specific colors. How can we reconcile the color-luminance differences in the blobs and interblobs (respectively) with corresponding differences in spatial frequency tuning in the blobs and interblobs?

One clue is that one-fourth of the blob cells respond best to luminance variations (Livingstone and Hubel, 1984); presumably these are among the cells taking up DG in response to lowspatial-frequency, achromatic gratings. Presumably some of the "color" cells will also respond to low-spatial-frequency luminance variations and thus contribute to the DG response.

Another clue is that the color and luminance systems themselves are sensitive to different ranges of spatial frequency. Sensitivity to color variations is maximal at low spatial frequencies, and sensitivity to luminance variations peaks higher. This has been shown both psychophysically (Van der Horst and Bouman, 1969; Granger and Heurtley, 1973; Watanabe et al., 1976) in the human, and physiologically (De Valois et al., 1977; Hicks et al., 1983; Thorell et al., 1984) in the Old World primate. Though no one can say for certain why such a state has evolved, it is true that chromatic aberration distorts high-spatial-frequency information in the color domain; this may rationalize the lack of high-spatial-frequency sensitivity to color variations. Very low spatial frequency information in the color domain is presumably useful in identifying and differentiating large objects of differing color. At any rate, the fact that the color system is biased towards low spatial frequencies matches the DG data showing that low-spatial-frequency luminance and color sensitivity is shared in the blobs.

With differences in both color and spatial frequency tuning existing between the blobs and interblobs, one might well wonder which is the primary function, and which the elaboration 
added on later. Consider the following idea, suggested initially by John Allman. The color vision of the owl monkey is very poor, almost negligible (Jacobs, 1977), yet it has prominent cytochrome oxidase blobs (Horton, 1984; Tootell et al., 1985). It would thus be of interest to see whether there are differences in spatial frequency tuning between blob and interblob regions of the owl monkey. Such a finding (coupled with a presumed lack of color sensitivity in the blobs) would imply that perception of the differences in spatial frequency tuning between blob and interblob regions is the primary function, onto which colorluminance sensitivity differences were elaborated.

\section{References}

Albrecht, D. G. (1978) Analysis of visual form. Doctoral dissertation, University of California, Berkeley, CA.

Albrecht, D. G., R. L. De Valois, and L. G. Thorell (1980) Visual cortical neurons: Are hars or gratings the optimal stimuli? Science 207: 88-90.

Blakemore, C., and F. W. Campbell (1969) On the existence of neurones in the human visual selectively sensitive to the orientation and size of retinal images. J. Physiol. (Lond.) 213: 157-174.

Blasdel, G. G., and D. Fitzpatrick (1984) Physiological organization of layer 4 in macaque striate cortex. J. Neurosci. 4: 880-895.

Braddick, O., F. W. Campbell, and J. Atkinson (1978) Channels in vision: Basic aspects. In Handbook of Sensory Physiology, Vol. 7, R. Held, H. W. Leibowitz, and H.-L. Teuber, eds., pp. 3-38, SpringerVerlag, Berlin.

Campbell, F. W., and J. J. Kulikowski (1966) Orientation selectivity of the human visual system. J. Physiol. (Lond.) 217: 437-445.

Campbell, F. W., and J. G. Robson (1968) Application of Fourier analysis to the visibility of gratings. J. Physiol. (Lond.) 197: 551-566.

Daugman, J. C. (1980) Two-dimensional spectral analysis of cortical receptive field profiles. Vision Res. 20:847-856.

de Monasterio, F. M., and P. Gouras (1975) Functional properties of ganglion cells of the rhesus monkey retina. J. Physiol. (Lond.) 251: 167-196.

Derrington, A. M., and P. Lennie (1984) Spatial and temporal contrast sensitivities of neurons in lateral geniculate nucleus of macaque. $J$. Physiol. (Lond.) 357: 219-240.

De Valois, R. L., K. K. De Valois, J. Ready, and H. von Blanckensee (1975) Spatial frequency tuning of macaque striate cortex cells. Res. Vision Ophthalmol., p. 16.

De Valois, R. L., D. M. Snodderly, Jr., E. W. Yund, and N. K. Hepler (1977) Response of macaque lateral geniculate cells to luminance and color figures. Sens. Processes $1:$ 244-259.

De Valois, R. L., D. G. Albrecht, and L. G. Thorell (1982) Spatial frequency selectivity of cells in macaque visual cortex. Vision Res. 22: 545-559.

De Valois, R. L., L. G. Thorell, and D. G. Albrecht (1985) Periodicity of striate-cortex-cell receptive fields. J. Opt. Soc. Am. A2: 1115-1123.

Dow, B. M., A. Z. Snyder, R. G. Vautin, and R. Bauer (1981) Magnification factor and receptive field size in foveal striate cortex of monkey. Exp. Brain Res. 44: 213-228.

Fitzpatrick, D., J. S. Lund, and G. G. Blasdel (1985) Intrinsic connections of macaque striate cortex: Afferent and efferent connections of lamina 4C. J. Neurosci. 5: 3329-3349.

Gilinski, A. S. (1968) Orientation-specific effects of patterns of adapting light on visual acuity. J. Opt. Soc. Am. 58: 13-18.

Ginsburg, A. P., D. W. Evans, M. W. Cannon, C. Owlsey, and P. Mulvanny (1984) Large-sample norm for contrast sensitivity. Am. J. Optom. Physiol. Optics 61: 80-84.

Granger, E. M., and J. C. Heurtley (1973) Visual chromaticity-modulation transfer function. J. Opt. Soc. Am. 63: 1173-1174.

Hendrickson, A. E., J. A. Movshon, H. M. Eggers, M. S. Gizzi, R. G. Boothe, and L. Kiorpes (1987) Effects of early unilateral blur on the macaque's visual system. II. Anatomical observations. J. Neurosci. 7: 1327-1339.

Hicks, T. P., B. B. Lee, and T. R. Vidyasagar (1983) The responses of cells in macaque lateral geniculate nucleus to sinusoidal gratings. J. Physiol. (Lond.) 337: 183-200.

Horton, J. C. (1984) Cytochrome oxidase patches; a new cytoarchi- tectonic feature of monkey visual cortex. Phil. Trans. R. Soc. Lond. [Biol.] 304: 199-253.

Horton, J. C., and D. H. Hubel (1981) Regular patchy distribution of cytochrome oxidase staining in primary visual cortex of macaque monkey. Nature 292: 762-764.

Hubel, D. H., and M. S. Livingstone (1987) Segregation of form, color, and stereopsis in primate area 18. J. Neurosci. 7: 3378-3415.

Hubel, D. H., and T. N. Wiesel (1972) Laminar and columnar distribution of geniculo-cortical fibers in the macaque monkey. J. Comp. Neurol. 158: 267-294.

Hubel, D. H., and T. N. Wiesel (1974) Uniformity of monkey striate cortex: A parallel relationship between field scatter and magnification factor. J. Comp. Neurol. 158: 295-306.

Humphrey, A. L., and A. E. Hendrickson (1983) Background and stimulus-induced patterns of high metabolic activity in the visual cortex (area 17) of the squirrel and macaque monkey. J. Neurosci. 3 . 345-358.

Jacobs, G. H. (1977) Visual capacities of the owl monkey (Aotus trivirgatus): I. Spectral sensitivity and color vision. Vision Res. 17: 811-820.

Kabrinsky, M. (1966) A Proposed Model for Visual Information Processing in the Human Brain, U. Illinois Press, Urbana, IL.

Kaplan, E., and R. M. Shapley (1982) X and Y cells in the lateral geniculate nucleus of macaque monkeys. J. Physiol. (Lond.) 330:125 143.

Kiorpes, L., R. G. Boothe, A. E. Hendrickson, J. A. Movshon, H. M. Eggers, and M. S. Gizzi (1987) Effects of early unilateral blur on the macaque's visual system. I. Behavioral observations. J. Neurosci. 7: $1318-1326$.

Klein, S. A., and D. M. Levi (1985) Hyperacuity thresholds of 1.0 second: Theoretical predictions and empirical validation. J. Opt. Soc. Am. A2: 1170-1 190.

Kulikowski, J. J., and P. O. Bishop (1981) Linear analysis of the responses of simple cells in the cat visual cortex. Exp. Brain Res. 44: $386-400$.

Livingstone, M. S., and D. H. Hubel (1984) Anatomy and physiology of a color system in the primate visual cortex. J. Neurosci. 4: 309356.

Lund, J. S. (1973) Organization of neurons in the visual cortex, area 17, of the monkey (Macaca mulatta). J. Comp. Neurol. 147: 455496.

Maffei, L., and A. Fiorentini (1973) The visual cortex as a spatial frequency analyzer. Vision Res. 13: 1255-1267.

Marcelja, S. (1980) Mathematical description of the responses of simple cortical cells. J. Opt. Soc. Am. 70: 1297-1300.

Michaels, C. R. (1986) Functional and morphological identification of double and single opponent color cells in layer IVCb of the monkey's striate cortex. Soc. Neurosci. Abstr. 12: 1497.

Michaels, C. R. (1987) Double opponent color cells in layer IVCb project to the blobs in monkey striate cortex. Inv. Ophthalmol. Vis. Sci. (Suppl.) 28: 196.

Movshon, J. A., I. D. Thompson, and D. J. Tolhurst (1978a) Spatial summation in the receptive fields of simple cells in the cat's striate cortex. J. Physiol. (Lond.) 283: 53-77.

Movshon, J. A., I. D. Thompson, and D. J. Tolhurst (1978b) Receptive field organization of complex cells in the cat's striate cortex. J. Physiol. (Lond.) 283: 79-99.

Movshon, J. A., I. D. Thompson, and D. J. Tolhurst (1978c) Spatial and temporal contrast sensitivity of neurones in areas 17 and 18 of the cat's visual cortex. J. Physiol. (Lond.) 283: 101-120.

Movshon, J. A., H. M. Eggers, M. S. Gizzi, A. E. Hendrickson, L. Kiorpes, and R. G. Boothe (1987) Effects of early unilateral blur on the macaque's visual system. III. Physiological observations. I. Neurosci. 7: 1340-1351.

Mulliken, W. H., J. P. Jones, and L. A. Palmer (1984) Periodic simple cells in cat area 17. J. Neurophysiol. 52: 372-387.

Perry, V. H., R. Oehler, and A. Cowey (1984) Retinal ganglion cells that project to the dorsal lateral geniculate nucleus in the macaque monkey. Neuroscience 12: 1101-1123.

Poggio, G. F., R. W. Doty, Jr., and W. H. Talbot (1977) Foveal striate cortex of behaving monkey: Single neuron responses to square wave grating during fixation of gaze. J. Neurophysiol. 40: 1369-1391.

Robson, J. G., and N. Graham (1981) Probability summation and regional variations in contrast sensitivity curves across the visual field. Vision Res. 21: 408-418. 
Rovamo, J., and V. Virsu (1979) An estimation and application of the human cortical magnification factor. Exp. Brain Res. 37: 495510.

Schiller, P. H., B. L. Finlay, and S. F. Volman (1976) Quantitative studies of single-cell properties in monkey striate cortex. III. Spatial frequency. J. Neurophysiol. 39: 1334-1351.

Shapley, R., and P. Lennie (1985) Spatial frequency analysis in the visual system. Annu. Rev. Neurosci. 8: 547-583.

Sherman, S. M., R. A. Schumer, and J. A. Movshon (1984) Functional cell classes in the macaque's LGN. Soc. Neurosci. Abstr. 10: 296.

Silverman, M. S. (1984) Deoxyglucose and electrophysiological evidence for spatial frequency columns in cat striate cortex. Doctoral disscrtation, University of California, San Francisco, CA

Stork, D. G., J. Z. Levinson, D. G. Albrecht, R. L. De Valois, and L. G. Thorell (1982) Receptive fields and the optimal stimulus. Science 216: 204-205.

Switkes, G., R. B. H. Tootell, M. S. Silverman, and R. L. De Valois (1986) Picture processing techniques applied to autoradiographic studies of visual cortex. J. Neurosci. Methods 15: 269-280.

Thorell, L. G., R. L. De Valois, and D. G. Albrecht (1984) Spatial mapping of monkey VI cells with pure color and luminance stimuli. Vision Res. 24: 751-769.

Tootell, R. B. H., and G. G. Blasdel (1987) Demonstration of a spatial frequency architecture in macaque striate cortex with voltage-sensitive dyes. Soc. Neurosci. Abstr. 13: 2.

Tootell, R. B. H., M. S. Silverman, E. Switkes, and R. L. De Valois (1981) Spatial frequency columns in primary visual cortex. Science 214: 813-815.

Tootell, R. B. H., M. S. Silverman, E. Switkes, and R. L. De Valois (1982) The organization of cortical modules in primate striate cortex. Soc. Neurosci. Abstr. 8: 707.

Tootell, R. B. H., S. L. Hamilton, and M. S. Silverman (1985) Topography of cytochrome oxidase activity in owl monkey cortex. J. Neurosci. 5: 2786-2800.

Tootell, R. B. H., S. L. Hamilton, M. S. Silverman, and E. Switkes (1988a) Functional anatomy of macaque striate cortex. I. Ocular dominance, binocular interactions, and baseline conditions. J. Neurosci. 8: $1500-1530$.
Tootell, R. B. H., E. Switkes, M. S. Silverman, and S. L. Hamilton (1988b) Functional anatomy of macaque striate cortex. II. Retinotopic organization. J. Neurosci. 8: 1531-1568.

Tootell, R. B. H., M. S. Silverman, S. L. Hamilton, R. L. De Valois, and $E$. Switkes (1988c) Functional anatomy of macaque striate cortex. III. Color. J. Neurosci. 8: 1569-1593.

Tootell, R. B. H., S. L. Hamilton, and E. Switkes (1988d) Functional anatomy of macaque striate cortex. IV. Contrast and magno-parvo streams. J. Neurosci. 8: 1594-1609.

Van der Horst, G. J. C., and M. A. Bouman (1969) Spatio-temporal chromaticity discrimination. J. Opt. Soc. Am. 59: 1482-1488.

Van Essen, D. C., W. T. Newsome, and J. H. R. Maunsell (1984) The visual field representation in striate cortex of the macaque monkey: Asymmetries, anisotropies and individual variability. Vision Res. 24: 429-448.

Watanabe, A., H. Sakata, and H. Isono (1976) Chromatic spatial sine wave response of the human visual system. NHK Lab Note 198: 110 .

Watson, A. B. (1983) Detection and recognition of simple spatial forms. In Physical and Biological Processing of Images, O. J. Braddick and A. C., eds., pp. 100-114, Springer-Verlag, New York.

Watson, A. B., and A. J. Ahumada (1983) A model of spatial contrast vision. Inv. Ophthalmol. Vis. Sci. (Suppl.) 24: 47.

Watson, A. B. and J. G. Robson (1981) Discrimination at threshold: Labelled detectors in human vision. Vision Res. 21: 1115-1122.

Wertheim, T. (1894) Uber die indirekte Sehscharfe. Z. Psychol. Physiol. Sinnesorg. 7: 172-189.

Westheimer, G. (1964) Pupil size and visual resolution. Vision Res. 4: $39-45$.

Wilson, H. R. (1985) Discrimination of contour curvature: Data and theory. J. Opt. Soc. Am. A2: 1191-1199.

Wilson, H. R. (1988) Responses of spatial mechanisms can explain hyperacuity. Vision Res. (in press)

Wilson, H. R., and D. J. Gelb (1984) Modified line-element theory for spatial-frequency discrimination. J. Opt. Soc. Am. 73: 1684-1690. 